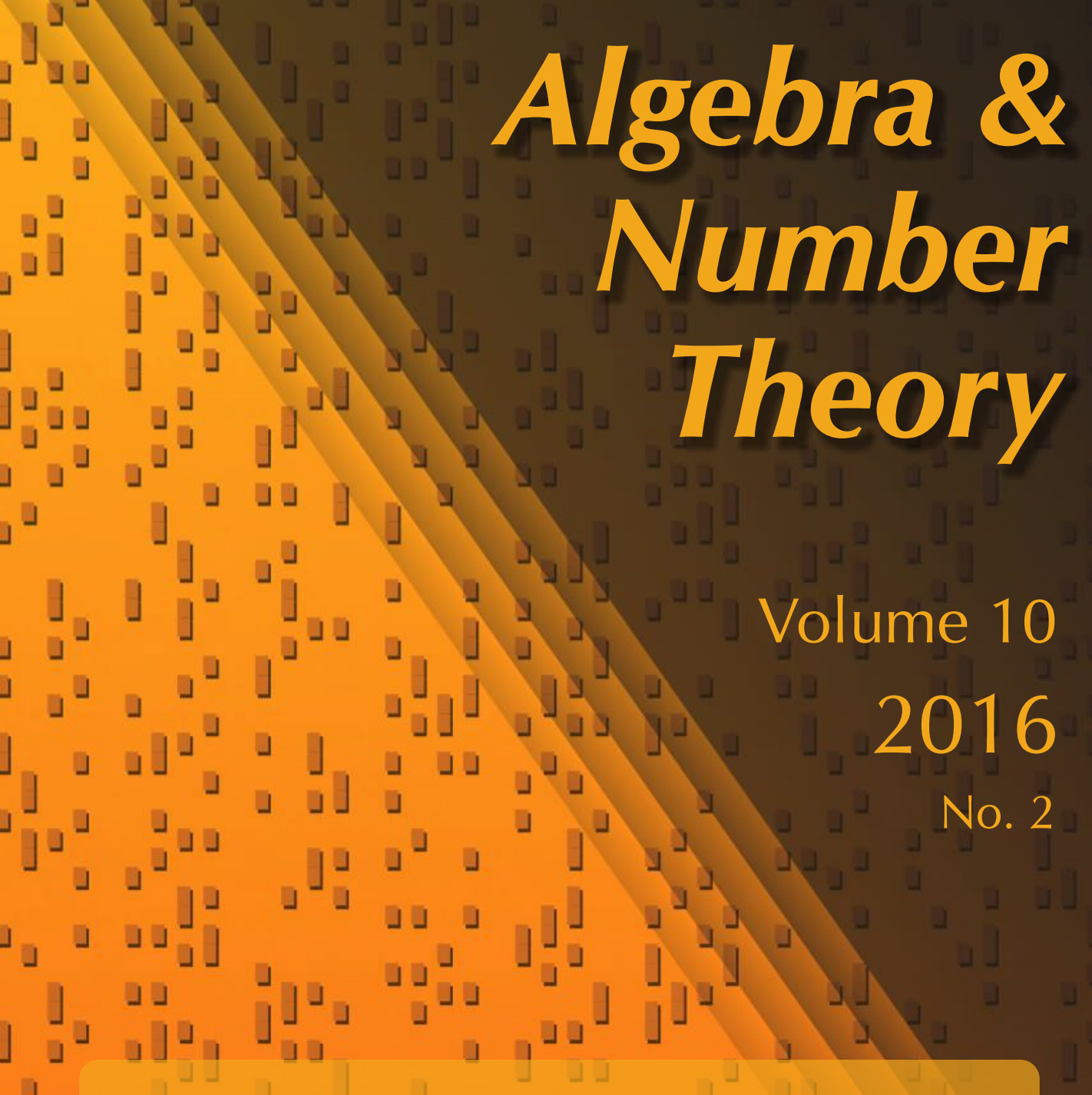

Kummer theory for-Drinfeld modules

Richard Pink

ل

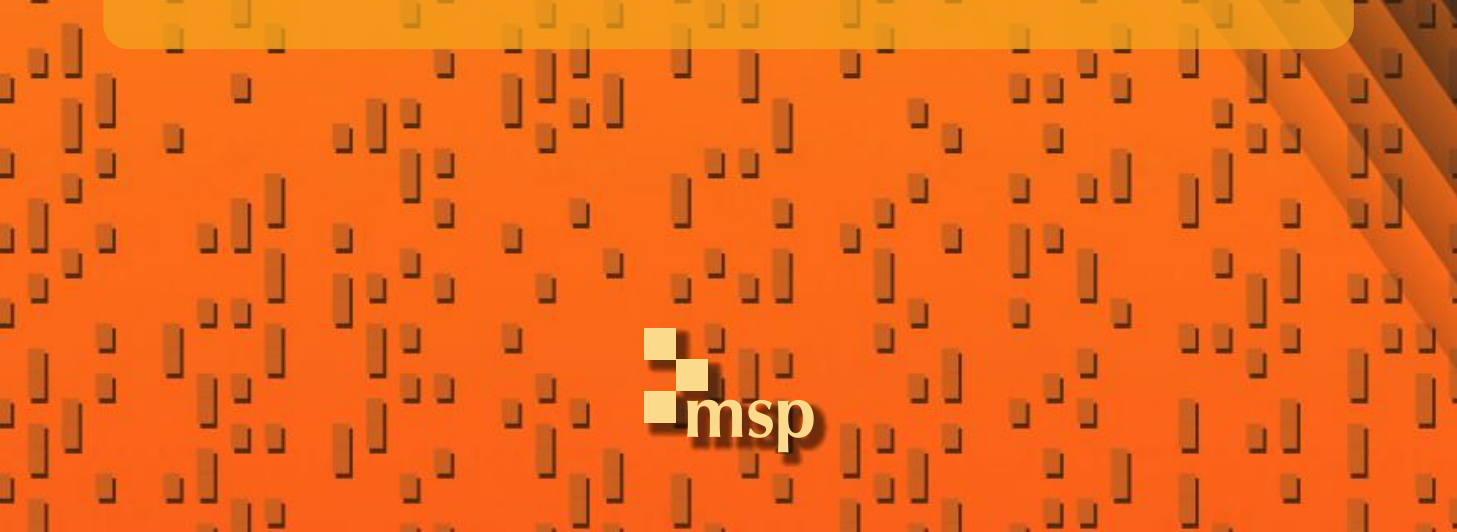




\title{
Kummer theory for Drinfeld modules
}

\author{
Richard Pink
}

Let $\varphi$ be a Drinfeld $A$-module of characteristic $\mathfrak{p}_{0}$ over a finitely generated field $K$. Previous articles determined the image of the absolute Galois group of $K$ up to commensurability in its action on all prime-to- $\mathfrak{p}_{0}$ torsion points of $\varphi$, or equivalently, on the prime-to- $\mathfrak{p}_{0}$ adelic Tate module of $\varphi$. In this article we consider in addition a finitely generated torsion free $A$-submodule $M$ of $K$ for the action of $A$ through $\varphi$. We determine the image of the absolute Galois group of $K$ up to commensurability in its action on the prime-to- $\mathfrak{p}_{0}$ division hull of $M$, or equivalently, on the extended prime-to- $\mathfrak{p}_{0}$ adelic Tate module associated to $\varphi$ and $M$.

1. Introduction

2. Extended Tate modules 217

3. Reduction steps 220

4. Previous results on Galois groups 223

5. The primitive case 227

6. The general case 231

References 234

\section{Introduction}

Let $F$ be a finitely generated field of transcendence degree 1 over the prime field $\mathbb{F}_{p}$ of characteristic $p>0$. Let $A$ be the ring of elements of $F$ which are regular outside a fixed place $\infty$ of $F$. Let $K$ be another field that is finitely generated over $\mathbb{F}_{p}$, and let $K^{\text {sep }}$ be a separable closure of $K$. Write $\operatorname{End}\left(\mathbb{G}_{a, K}\right)=K[\tau]$ with $\tau(x)=x^{p}$. Let $\varphi: A \rightarrow K[\tau], a \mapsto \varphi_{a}$ be a Drinfeld $A$-module of rank $r \geqslant 1$ and characteristic $\mathfrak{p}_{0}$. Then either $\mathfrak{p}_{0}$ is the zero ideal of $A$ and $\varphi$ is said to have generic characteristic; or $\mathfrak{p}_{0}$ is a maximal ideal of $A$ and $\varphi$ is said to have special characteristic.

For brevity we call any maximal ideal of $A$ a prime of $A$. For any prime $\mathfrak{p} \neq \mathfrak{p}_{0}$ of $A$ the $\mathfrak{p}$-adic Tate module $T_{\mathfrak{p}}(\varphi)$ is a free module of rank $r$ over the completion $A_{\mathfrak{p}}$, endowed with a continuous action of the $\operatorname{Galois} \operatorname{group} \operatorname{Gal}\left(K^{\mathrm{sep}} / K\right)$. The prime-to$\mathfrak{p}_{0}$ adelic Tate module $T_{\mathrm{ad}}(\varphi)=\prod_{\mathfrak{p} \neq \mathfrak{p}_{0}} T_{\mathfrak{p}}(\varphi)$ is then a free module of rank $r$ over 
$A_{\text {ad }}=\prod_{\mathfrak{p} \neq \mathfrak{p}_{0}} A_{\mathfrak{p}}$ carrying a natural action of Galois. This action corresponds to a continuous homomorphism

$$
\mathrm{Gal}\left(K^{\mathrm{sep}} / K\right) \rightarrow \operatorname{Aut}_{A_{\mathrm{ad}}}\left(T_{\mathrm{ad}}(\varphi)\right) \cong \mathrm{GL}_{r}\left(A_{\mathrm{ad}}\right) .
$$

Its image $\Gamma_{\text {ad }}$ was determined up to commensurability in [Pink and Rütsche 2009] and [Devic and Pink 2012]; for special cases see Theorems 1.6 and 4.4 below.

Let $M \subset K$ be a finitely generated torsion free $A$-submodule of rank $d$ for the action of $A$ through $\varphi$. Then there is an associated prime-to- $p_{0}$ adelic Tate module $T_{\mathrm{ad}}(\varphi, M)$, which is a free module of rank $r+d$ over $A_{\mathrm{ad}}$ carrying a natural continuous action of $\operatorname{Gal}\left(K^{\mathrm{sep}} / K\right)$. This module lies in a natural Galois equivariant short exact sequence

$$
0 \longrightarrow T_{\mathrm{ad}}(\varphi) \longrightarrow T_{\mathrm{ad}}(\varphi, M) \longrightarrow M \otimes_{A} A_{\mathrm{ad}} \longrightarrow 0 .
$$

Define $\Gamma_{\mathrm{ad}, M}$ as the image of the associated continuous homomorphism

$$
\operatorname{Gal}\left(K^{\text {sep }} / K\right) \rightarrow \operatorname{Aut}_{A_{\text {ad }}}\left(T_{\text {ad }}(\varphi, M)\right) \cong \mathrm{GL}_{r+d}\left(A_{\text {ad }}\right) .
$$

Then the restriction to $T_{\mathrm{ad}}(\varphi)$ induces a surjective homomorphism $\Gamma_{\mathrm{ad}, M} \rightarrow \Gamma_{\mathrm{ad}}$, whose kernel we denote by $\Delta_{\mathrm{ad}, M}$. Since the action on $M \otimes_{A} A_{\mathrm{ad}}$ is trivial, there is a natural inclusion

$$
\Delta_{\mathrm{ad}, M} \hookrightarrow \operatorname{Hom}_{A}\left(M, T_{\mathrm{ad}}(\varphi)\right) .
$$

Any splitting of the sequence (1.2) induces an inclusion into the semidirect product

$$
\Gamma_{\mathrm{ad}, M} \hookrightarrow \Gamma_{\mathrm{ad}} \ltimes \operatorname{Hom}_{A}\left(M, T_{\mathrm{ad}}(\varphi)\right) .
$$

The aim of this article is to describe these subgroups up to commensurability.

In general the shape of these Galois groups is affected by the endomorphisms of $\varphi$ over $K^{\text {sep }}$, and in special characteristic also by the endomorphisms of the restrictions of $\varphi$ to all subrings of $A$. Any general results therefore involve further definitions and notation. In this introduction we avoid these and mention only a special case; the general case is addressed by Theorems 5.1, 6.6 and 6.7. Parts (a) and (b) of the following result can be found as [Pink and Rütsche 2009, Theorem 0.1] and [Devic and Pink 2012, Theorem 1.1], respectively, and part (c) is a special case of Theorem 5.1 below:

Theorem 1.6. Assume that $\operatorname{End}_{K^{\operatorname{sep}}}(\varphi)=A$, and in special characteristic also that $\operatorname{End}_{K^{\operatorname{sep}}}(\varphi \mid B)=A$ for every integrally closed infinite subring $B \subset A$.

(a) If $\varphi$ has generic characteristic, then $\Gamma_{\mathrm{ad}}$ is open in $\mathrm{GL}_{r}\left(A_{\mathrm{ad}}\right)$.

(b) If $\varphi$ has special characteristic, then $\Gamma_{\mathrm{ad}}$ is commensurable with $\overline{\left\langle a_{0}\right\rangle} \cdot \mathrm{SL}_{r}\left(A_{\mathrm{ad}}\right)$ for some central element $a_{0} \in A$ that generates a positive power of $\mathfrak{p}_{0}$.

(c) The inclusions (1.4) and (1.5) are both open. 
The method used to prove Theorem 1.6(c) and its generalizations is an adaptation of the Kummer theory for semiabelian varieties from Ribet [1979] and predecessors. The main ingredients are the above mentioned descriptions of $\Gamma_{\mathrm{ad}}$ and Poonen's tameness result [1995] concerning the structure of $K$ as an $A$-module via $\varphi$. A standard procedure would be to first prove corresponding results for $\mathfrak{p}$-division points for almost all primes $\mathfrak{p} \neq \mathfrak{p}_{0}$ of $A$, and for $\mathfrak{p}$-power division points for all $\mathfrak{p} \neq \mathfrak{p}_{0}$, and then to combine these individual results by taking products, as in [Ribet 1979; Chi and Li 2001; Li 2001; Pink and Rütsche 2009; Devic and Pink 2012; Häberli 2011]. Instead, we have found a shorter way by doing everything adelically from the start. The core of the argument is the proof of Lemma 5.3. Therein we avoid the explicit use of group cohomology by trivializing an implicit 1-cocycle with the help of a suitable central element of $\Gamma_{\mathrm{ad}}$. On first reading the readers may want to restrict their attention to the case of Theorem 1.6, which requires only Section 2, a little from Section 4, and Section 5 with simplifications, avoiding Sections 3 and 6 entirely. Some of this was worked out in [Häberli 2011]. Our results generalize those of [Chi and Li 2001] and [Li 2001].

The notation and the assumptions of this introduction remain in force throughout the article. For the general theory of Drinfeld modules see [Drinfeld 1974; Deligne and Husemöller 1987; Hayes 1979; Goss 1996].

\section{Extended Tate modules}

Following the usual convention in commutative algebra we let $A_{\left(\mathfrak{p}_{0}\right)} \subset F$ denote the localization of $A$ at $\mathfrak{p}_{0}$; this is equal to $F$ if and only if $\varphi$ has generic characteristic. Observe that there is a natural isomorphism of $A$-modules

$$
A_{\left(\mathfrak{p}_{0}\right)} / A \cong \bigoplus_{\mathfrak{p} \neq \mathfrak{p}_{0}} F_{\mathfrak{p}} / A_{\mathfrak{p}}
$$

where the product is extended over all maximal ideals $\mathfrak{p} \neq \mathfrak{p}_{0}$ of $A$ and where $F_{\mathfrak{p}}$ and $A_{\mathfrak{p}}$ denote the corresponding completions of $F$ and $A$. This induces a natural isomorphism for the prime-to- $\mathfrak{p}_{0}$ adelic completion of $A$ :

$$
A_{\mathrm{ad}}:=\operatorname{End}_{A}\left(A_{\left(\mathfrak{p}_{0}\right)} / A\right) \cong \prod_{\mathfrak{p} \neq \mathfrak{p}_{0}} A_{\mathfrak{p}}
$$

As a consequence, for any torsion $A$-module $X$ that is isomorphic to $\left(A_{\left(\mathfrak{p}_{0}\right)} / A\right)^{\oplus n}$ for some integer $n$, the construction

$$
T(X):=\operatorname{Hom}_{A}\left(A_{\left(\mathfrak{p}_{0}\right)} / A, X\right)
$$

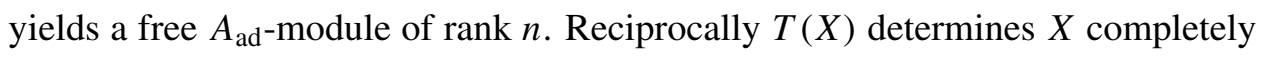
up to a natural isomorphism $X \cong T(X) \otimes_{A_{\text {ad }}}\left(A_{\left(\mathfrak{p}_{0}\right)} / A\right)$. Thus any $A$-linear group 
action on $X$ determines and is determined by the corresponding $A_{\text {ad }}$-linear group action on $T(X)$. Moreover, with

$$
T_{\mathfrak{p}}(X):=\operatorname{Hom}_{A}\left(F_{\mathfrak{p}} / A_{\mathfrak{p}}, X\right)
$$

the decompositions (2.1) and (2.2) induce a decomposition

$$
T(X) \cong \prod_{\mathfrak{p} \neq \mathfrak{p}_{0}} T_{\mathfrak{p}}(X) .
$$

This will give a concise way of defining the $\mathfrak{p}$-adic and adelic Tate modules associated to the given Drinfeld module $\varphi$.

We view $K^{\text {sep }}$ as an $A$-module with respect to the action $A \times K^{\text {sep }} \rightarrow K^{\text {sep }}$, $(a, x) \mapsto \varphi_{a}(x)$ and are interested in certain submodules. One particular submodule is $K$. Let $M$ be a finitely generated torsion free $A$-submodule of rank $d \geqslant 0$ contained in $K$. Then the prime-to-p p $_{0}$ division hull of $M$ in $K^{\text {sep }}$ is the $A$-submodule

$$
\operatorname{Div}_{K^{\text {sep }}}^{\left(\mathfrak{p}_{0}\right)}(M):=\left\{x \in K^{\text {sep }} \mid \exists a \in A \backslash \mathfrak{p}_{0}: \varphi_{a}(x) \in M\right\} .
$$

Let $\operatorname{Div}_{K}^{\left(\mathfrak{p}_{0}\right)}(M)$ denote the intersection of $\operatorname{Div}_{K^{\text {sep }}}^{\left(\mathfrak{p}_{0}\right)}(M)$ with $K$. For later use we recall the following result proved in [Poonen 1995, Lemma 5] when $K$ is a global field and $\varphi$ has generic characteristic, and in [Wang 2001] in general:

Theorem 2.7. $\left[\operatorname{Div}_{K}^{\left(\mathfrak{p}_{0}\right)}(M): M\right]$ is finite.

As a special case of the above, the prime-to- $\mathfrak{p}_{0}$ division hull of the zero module $\operatorname{Div}_{K^{\text {sep }}}^{\left(\mathfrak{p}_{0}\right)}(\{0\})$ is the module of all prime-to- $\mathfrak{p}_{0}$ torsion points of $\varphi$ in $K^{\text {sep }}$. By direct calculation, which we leave to the reader, one proves:

Proposition 2.8. There is a natural short exact sequence of A-modules

$$
0 \longrightarrow \operatorname{Div}_{K^{\text {sep }}}^{\left(\mathfrak{p}_{0}\right)}(\{0\}) \longrightarrow \operatorname{Div}_{K^{\text {sep }}}^{\left(\mathfrak{p}_{0}\right)}(M) \longrightarrow M \otimes_{A} A_{\left(\mathfrak{p}_{0}\right)} \longrightarrow 1,
$$

where the map on the right hand side is described by $x \mapsto \varphi_{a}(x) \otimes \frac{1}{a}$ for any $a \in A \backslash \mathfrak{p}_{0}$ satisfying $\varphi_{a}(x) \in M$.

Dividing by $M$, the exact sequence from Proposition 2.8 yields a natural short exact sequence of $A$-modules

$$
0 \longrightarrow \operatorname{Div}_{K^{\text {sep }}}^{\left(\mathfrak{p}_{0}\right)}(\{0\}) \longrightarrow \operatorname{Div}_{K^{\text {sep }}}^{\left(\mathfrak{p}_{0}\right)}(M) / M \longrightarrow M \otimes_{A}\left(A_{\left(\mathfrak{p}_{0}\right)} / A\right) \longrightarrow 0 .
$$

By the general theory of Drinfeld modules (see [Drinfeld 1974, Proposition 2.2]) the module on the left is isomorphic to $\left(A_{\left(\mathfrak{p}_{0}\right)} / A\right)^{\oplus r}$, where $r$ is the rank of $\varphi$. Using the functor $T$ from (2.3), the prime-to- $\mathfrak{p}_{0}$ adelic Tate module of $\varphi$ can be described canonically as

$$
T_{\mathrm{ad}}(\varphi):=T\left(\operatorname{Div}_{K^{\operatorname{sep}}}^{\left(\mathfrak{p}_{0}\right)}(\{0\})\right)
$$


and is a free $A_{\text {ad }}$-module of rank $r$. Since $M$ is a projective $A$-module of rank $d$, the module on the right of (2.9) is isomorphic to $\left(A_{\left(\mathfrak{p}_{0}\right)} / A\right)^{\oplus d}$, and together it follows that the module in the middle is isomorphic to $\left(A_{\left(\mathfrak{p}_{0}\right)} / A\right)^{\oplus(r+d)}$. The extended prime-to- $\mathfrak{p}_{0}$ adelic Tate module of $\varphi$ and $M$

$$
T_{\mathrm{ad}}(\varphi, M):=T\left(\operatorname{Div}_{K^{\text {sep }}}^{\left(\mathfrak{p}_{0}\right)}(M) / M\right)
$$

is therefore a free $A_{\text {ad }}$-module of rank $r+d$. Moreover, the exact sequence (2.9) yields a natural short exact sequence of $A_{\text {ad }}$-modules

$$
0 \longrightarrow T_{\mathrm{ad}}(\varphi) \longrightarrow T_{\mathrm{ad}}(\varphi, M) \longrightarrow 0 .
$$

All this decomposes uniquely as $T_{\mathrm{ad}}(\varphi)=\prod_{\mathfrak{p} \neq \mathfrak{p}_{0}} T_{\mathfrak{p}}(\varphi)$ etc. as in (2.5).

By construction there is a natural continuous action of the Galois group

$$
\operatorname{Gal}\left(K^{\mathrm{sep}} / K\right)
$$

on all modules and arrows in Proposition 2.8 and in (2.9). This induces a continuous action on the short exact sequence (2.12), which in turn determines the former two by the following fact:

Proposition 2.13. The action of $\operatorname{Gal}\left(K^{\mathrm{sep}} / K\right)$ on $\operatorname{Div}_{K^{\text {sep }}}^{\left(\mathfrak{p}_{0}\right)}(M)$ is completely determined by the action on $T_{\mathrm{ad}}(\varphi, M)$.

Proof. For any $\sigma \in \operatorname{Gal}\left(K^{\text {sep }} / K\right)$ the endomorphism $x \mapsto \sigma(x)-x$ of $\operatorname{Div}_{K^{\text {sep }}}^{\left(\mathfrak{p}_{0}\right)}(M)$ is trivial on $M$, because that module is contained in $K$. Also, the image of this endomorphism is contained in $\operatorname{Div}_{K}^{\left(\mathfrak{p}_{0}\right)}(\{0\})$, because for any $a \in A \backslash \mathfrak{p}_{0}$ with $\varphi_{a}(x) \in M$ we have

$$
\varphi_{a}(\sigma(x)-x)=\sigma\left(\varphi_{a}(x)\right)-\varphi_{a}(x)=0 .
$$

Thus the endomorphism factors through a homomorphism

$$
\operatorname{Div}_{K^{\text {sep }}}^{\left(\mathfrak{p}_{0}\right)}(M) / M \longrightarrow \operatorname{Div}_{K^{\operatorname{sep}}}^{\left(\mathfrak{p}_{0}\right)}(\{0\}) .
$$

But by (2.9) the latter homomorphism is determined completely by the action of $\sigma$ on $\operatorname{Div}_{K}^{\left(\mathfrak{p}_{0}\right)}(M) / M$, and thus by the action of $\sigma$ on $T_{\mathrm{ad}}(\varphi, M)$, as desired.

Let $\Gamma_{\mathrm{ad}}$ and $\Gamma_{\mathrm{ad}, M}$ denote the images of $\operatorname{Gal}\left(K^{\mathrm{sep}} / K\right)$ acting on $T_{\mathrm{ad}}(\varphi)$ and $T_{\mathrm{ad}}(\varphi, M)$, as in (1.1) and (1.3). Restricting to $T_{\mathrm{ad}}(\varphi)$ induces a surjective homomorphism $\Gamma_{\mathrm{ad}, M} \rightarrow \Gamma_{\mathrm{ad}}$, and we define $\Delta_{\mathrm{ad}, M}$ by the short exact sequence

$$
1 \longrightarrow \Delta_{\mathrm{ad}, M} \longrightarrow \Gamma_{\mathrm{ad}, M} \longrightarrow \Gamma_{\mathrm{ad}} \longrightarrow 1 \text {. }
$$

For any $m \in M$ take an element $t \in T_{\mathrm{ad}}(\varphi, M)$ with image $m \otimes 1$ in $M \otimes_{A} A_{\text {ad }}$. Since any $\delta \in \Delta_{\mathrm{ad}, M}$ acts trivially on $T_{\mathrm{ad}}(\varphi)$, the difference $\delta(t)-t$ depends only 
on $\delta$ and $m$. Since $\delta$ also acts trivially on $M \otimes_{A} A_{\text {ad }}$, the difference lies in $T_{\text {ad }}(\varphi)$ and therefore defines a map

$$
\Delta_{\mathrm{ad}, M} \times M \longrightarrow T_{\mathrm{ad}}(\varphi), \quad(\delta, m) \mapsto\langle\delta, m\rangle:=\delta(t)-t .
$$

By direct calculation this map is additive in $\delta$ and $A$-linear in $m$. By the construction of $\Delta_{\mathrm{ad}, M}$ the adjoint of the pairing (2.15) is therefore a natural inclusion, already mentioned in (1.4):

$$
\Delta_{\mathrm{ad}, M} \hookrightarrow \operatorname{Hom}_{A}\left(M, T_{\mathrm{ad}}(\varphi)\right) .
$$

Let $R:=\operatorname{End}_{K}(\varphi)$ denote the endomorphism ring of $\varphi$ over $K$. This is an $A$-order in a finite dimensional division algebra over $F$ (see [Drinfeld 1974, Corollary to Proposition 2.4]). It acts naturally on $K$ and $K^{\text {sep }}$ and therefore on $\operatorname{Div}_{K^{\text {sep }}}^{\left(\mathfrak{p}_{0}\right)}(\{0\})$ and $T_{\text {ad }}(\varphi)$, turning the latter two into modules over $R_{\mathrm{ad}}:=R \otimes_{A} A_{\mathrm{ad}}$. As this action commutes with the action of $\Gamma_{\mathrm{ad}}$, it leads to an inclusion

$$
\Gamma_{\text {ad }} \subset \operatorname{Aut}_{R_{\text {ad }}}\left(T_{\text {ad }}(\varphi)\right) .
$$

The decomposition (2.2) induces a decomposition

$$
R_{\mathrm{ad}}=\prod_{\mathfrak{p} \neq \mathfrak{p}_{0}} R_{\mathfrak{p}},
$$

where $R_{\mathfrak{p}}:=R \otimes_{A} A_{\mathfrak{p}}$ acts naturally on $T_{\mathfrak{p}}(\varphi)$.

If $M$ is an $R$-submodule of $K$, then $R$ and hence $R_{\text {ad }}$ also act on $\operatorname{Div}_{K^{\text {sep }}}^{\left(\mathfrak{p}_{0}\right)}(M)$ and $T_{\mathrm{ad}}(\varphi, M)$, and these actions commute with the action of $\Gamma_{\mathrm{ad}, M}$. The inclusion (2.16) then factors through an inclusion

$$
\Delta_{\mathrm{ad}, M} \hookrightarrow \operatorname{Hom}_{R}\left(M, T_{\mathrm{ad}}(\varphi)\right) .
$$

Moreover, any $R$-equivariant splitting of the sequence (2.12) then induces an embedding into the semidirect product

$$
\Gamma_{\mathrm{ad}, M} \hookrightarrow \Gamma_{\mathrm{ad}} \ltimes \operatorname{Hom}_{R}\left(M, T_{\mathrm{ad}}(\varphi)\right) .
$$

\section{Reduction steps}

For use in Section 6 we now discuss the behavior of extended Tate modules and their associated Galois groups under isogenies and under restriction of $\varphi$ to subrings.

First consider another Drinfeld $A$-module $\varphi^{\prime}$ and an isogeny $f: \varphi \rightarrow \varphi^{\prime}$ defined over $K$. Recall that there exists an isogeny $g: \varphi^{\prime} \rightarrow \varphi$ such that $g \circ f=\varphi_{a}$ for some nonzero $a \in A$ (see [Drinfeld 1974, Corollary to Proposition 2.3]). From this it follows that $M^{\prime}:=f(M)$ is a torsion free finitely generated $A$-submodule of $K$ for the action of $A$ through $\varphi^{\prime}$. Thus $f$ induces $A_{\text {ad-linear maps from the modules in }}$ (2.12) to those associated to $\varphi^{\prime}$ and $M^{\prime}$. The existence of $g$ implies that these maps 
are inclusions of finite index. Together these maps yield a commutative diagram of $A_{\text {ad-modules with exact rows }}$

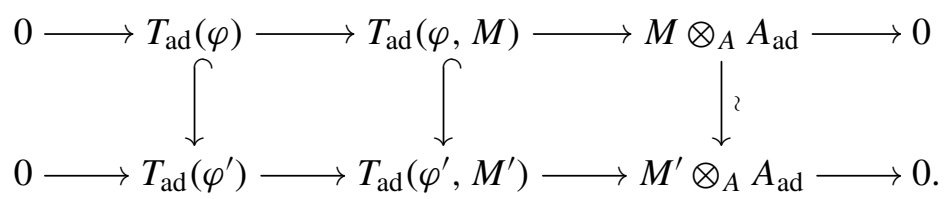

By construction all these maps are equivariant under $\operatorname{Gal}\left(K^{\mathrm{sep}} / K\right)$; hence the images of Galois in each column are canonically isomorphic. If we denote the analogues of the groups $\Delta_{\mathrm{ad}, M} \subset \Gamma_{\mathrm{ad}, M} \rightarrow \Gamma_{\mathrm{ad}}$ associated to $\varphi^{\prime}$ and $M^{\prime}$ by

$$
\Delta_{\mathrm{ad}, M^{\prime}}^{\varphi^{\prime}} \subset \Gamma_{\mathrm{ad}, M^{\prime}}^{\varphi^{\prime}} \rightarrow \Gamma_{\mathrm{ad}}^{\varphi^{\prime}},
$$

this means that we have a natural commutative diagram

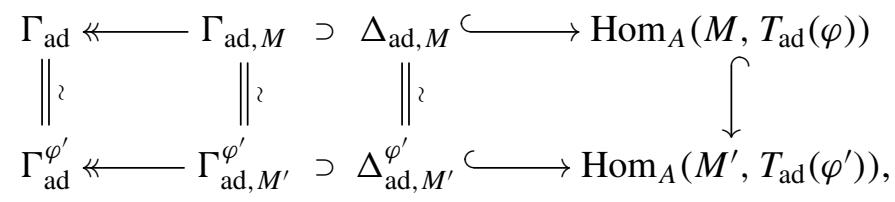

where the vertical arrow on the right hand side is an inclusion of finite index.

Next let $B$ be any integrally closed infinite subring of $A$. Then $A$ is a finitely generated projective $B$-module of some rank $s \geqslant 1$. The restriction $\psi:=\varphi \mid B$ is therefore a Drinfeld $B$-module of rank $r s$ over $K$, and the given $A$-module $M$ of rank $d$ becomes a $B$-module of rank $d s$. Moreover, since the characteristic of $\varphi$ is by definition the kernel of the derivative map $a \mapsto d \varphi_{a}$, the characteristic of $\psi$ is simply $\mathfrak{q}_{0}:=\mathfrak{p}_{0} \cap B$. In analogy to (2.2) we have

$$
B_{\text {ad }}:=\operatorname{End}_{B}\left(B_{\left(\mathfrak{q}_{0}\right)} / B\right) \cong \prod_{\mathfrak{q} \neq \mathfrak{q}_{0}} B_{\mathfrak{q}},
$$

where the product is extended over all maximal ideals $\mathfrak{q} \neq \mathfrak{q}_{0}$ of $B$. Thus

$$
A \otimes_{B} B_{\mathrm{ad}} \cong \prod_{\mathfrak{p} \nmid \mathfrak{q}_{0}} A_{\mathfrak{p}}
$$

is in a natural way a factor ring of $A_{\text {ad }}$. More precisely, it is isomorphic to $A_{\text {ad }}$ if the characteristic $\mathfrak{p}_{0}$ and hence $\mathfrak{q}_{0}$ is zero; otherwise it is obtained from $A_{\text {ad }}$ by removing the finitely many factors $A_{\mathfrak{p}}$ for all maximal ideals $\mathfrak{p} \neq \mathfrak{p}_{0}$ of $A$ above $\mathfrak{q}_{0}$. In particular we have a natural isomorphism $A \otimes_{B} B_{\text {ad }} \cong A_{\text {ad }}$ if and only if $\mathfrak{p}_{0}$ is the unique prime ideal of $A$ above $\mathfrak{q}_{0}$.

Proposition 3.5. The exact sequence (2.12) for $\psi$ and $M$ is naturally isomorphic to that obtained from the exact sequence (2.12) for $\varphi$ and $M$ by tensoring with 
$A \otimes_{B} B_{\mathrm{ad}}$ over $A_{\mathrm{ad}}$. In particular we have a commutative diagram with surjective vertical arrows

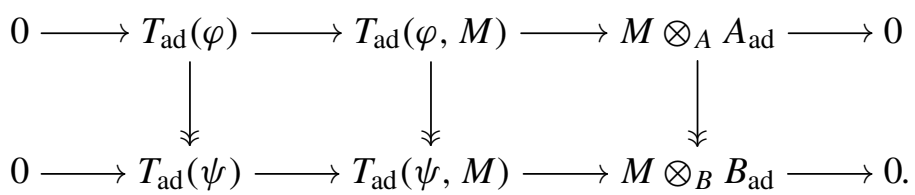

If $\mathfrak{p}_{0}$ is the only prime ideal of $A$ above $\mathfrak{q}_{0}$, the vertical arrows are isomorphisms.

Proof. According to (2.6) the prime-to- $\mathfrak{p}_{0}$ division hull of $M$ with respect to $\varphi$ and the prime-to- $\mathfrak{q}_{0}$ division hull of $M$ with respect to $\psi$ are

$$
\begin{aligned}
& \operatorname{Div}_{K^{\text {sep }}}^{\left(\mathfrak{p}_{0}\right)}(M):=\left\{x \in K^{\text {sep }} \mid \exists a \in A \backslash \mathfrak{p}_{0}: \varphi_{a}(x) \in M\right\}, \\
& \operatorname{Div}_{K^{\text {sep }}}^{\left(\mathfrak{q}_{0}\right)}(M):=\left\{x \in K^{\text {sep }} \mid \exists b \in B \backslash \mathfrak{q}_{0}: \psi_{b}(x) \in M\right\} .
\end{aligned}
$$

Here the latter is automatically contained in the former, because any $b \in B \backslash \mathfrak{q}_{0}$ with $\psi_{b}(x) \in M$ is by definition an element $a:=b \in A \backslash \mathfrak{p}_{0}$ with $\varphi_{a}(x) \in M$. Thus $\operatorname{Div}_{K^{\text {sep }}}^{\left(\mathfrak{q}_{0}\right)}(M) / M$ is the subgroup of all elements of $\operatorname{Div}_{K^{\text {sep }}}^{\left(\mathfrak{p}_{0}\right)}(M) / M$ that are annihilated by some element of $B \backslash \mathfrak{q}_{0}$. In other words, it is the subgroup of all prime-to- $\mathfrak{q}_{0}$ torsion with respect to $B$, or again, it is obtained from $\operatorname{Div}_{K^{\text {sep }}}^{\left(\mathfrak{p}_{0}\right)}(M) / M$ by removing the $\mathfrak{p}$-torsion for all maximal ideals $\mathfrak{p} \neq \mathfrak{p}_{0}$ of $A$ above $\mathfrak{q}_{0}$. In the same way $A \otimes_{B}\left(B_{\left(\mathfrak{q}_{0}\right)} / B\right)$ is isomorphic to the submodule of $A_{\left(\mathfrak{p}_{0}\right)} / A$ obtained by removing the $\mathfrak{p}$-torsion for all $\mathfrak{p} \mid \mathfrak{q}_{0}$. The same process applied to the exact sequence (2.9) therefore yields the analogue for $\psi$ and $M$. By definition the exact sequence (2.12) for $\psi$ and $M$ is obtained from this by applying the functor

$$
X \mapsto \operatorname{Hom}_{B}\left(B_{\left(\mathfrak{q}_{0}\right)} / B, X\right) \cong \operatorname{Hom}_{A}\left(A \otimes_{B}\left(B_{\left(\mathfrak{q}_{0}\right)} / B\right), X\right)
$$

analogous to (2.3). The total effect of this is simply to remove the $\mathfrak{p}$-primary factors for all $\mathfrak{p} \mid \mathfrak{q}_{0}$ from the exact sequence (2.12) for $\varphi$ and $M$, from which everything follows.

By construction the diagram in Proposition 3.5 is equivariant under $\operatorname{Gal}\left(K^{\mathrm{sep}} / K\right)$. It therefore induces a natural commutative diagram of Galois groups

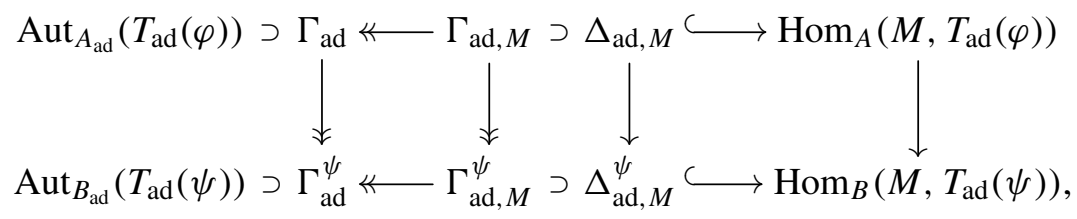

where the subgroups in the lower row are the analogues for $\psi$ and $M$ of those in the upper row. By construction the left two vertical arrows are surjective, and they are isomorphisms if $\mathfrak{p}_{0}$ is the only prime ideal of $A$ above $\mathfrak{q}_{0}$. In that case the rightmost vertical arrow is injective and it follows that the map $\Delta_{\mathrm{ad}, M} \rightarrow \Delta_{\mathrm{ad}, M}^{\psi}$ is 
an isomorphism as well. In general one can only conclude that $\Delta_{\mathrm{ad}, M}^{\psi}$ contains the image of $\Delta_{\mathrm{ad}, M}$. In any case the diagram (3.6) gives a precise way of determining $\Gamma_{\mathrm{ad}, M}^{\psi}$ from $\Gamma_{\mathrm{ad}, M}$.

\section{Previous results on Galois groups}

In this section we recall some previous results on the Galois group $\Gamma_{\mathrm{ad}}$. Its precise description up to commensurability depends on certain endomorphism rings. The endomorphism ring of a Drinfeld module of generic characteristic is always commutative, but in special characteristic it can be noncommutative. In the latter case it can grow on restricting $\varphi$ to a subring $B$ of $A$, and this effect can impose additional conditions on $\Gamma_{\text {ad }}$. The question of whether the endomorphism ring becomes stationary or grows indefinitely with $B$ depends on the following property:

Definition 4.1. We call a Drinfeld $A$-module of special characteristic over $K$ isotrivial if over $K^{\text {sep }}$ it is isomorphic to a Drinfeld $A$-module defined over a finite field.

The next definition is slightly ad hoc, but it describes particular kinds of Drinfeld modules to which we can reduce ourselves in all cases, allowing a unified treatment of Kummer theory later on.

Definition 4.2. We call the triple $(A, K, \varphi)$ primitive if the following conditions hold:

(a) $R:=\operatorname{End}_{K}(\varphi)$ is equal to $\operatorname{End}_{K}$ sep $(\varphi)$.

(b) The center of $R$ is $A$.

(c) $R$ is a maximal $A$-order in $R \otimes_{A} F$.

(d) If $\varphi$ is nonisotrivial of special characteristic, then for every integrally closed infinite subring $B \subset A$ we have $\operatorname{End}_{K^{\operatorname{sep}}}(\varphi \mid B)=R$.

(e) If $\varphi$ is isotrivial of special characteristic, then $A=\mathbb{F}_{p}\left[a_{0}\right]$ with $\varphi_{a_{0}}=\tau^{\left[k / \mathbb{F}_{p}\right]}$, where $k$ denotes the finite field of constants of $K$.

Proposition 4.3. Let $A^{\prime}$ denote the normalization of the center of $\operatorname{End}_{K^{\operatorname{sep}}}(\varphi)$.

(a) There exist a Drinfeld $A^{\prime}$-module $\varphi^{\prime}: A^{\prime} \rightarrow K^{\mathrm{sep}}[\tau]$ and an isogeny

$$
f: \varphi \rightarrow \varphi^{\prime} \mid A
$$

over $K^{\text {sep }}$ such that $A^{\prime}$ is the center of $\operatorname{End}_{K^{\operatorname{sep}}}\left(\varphi^{\prime}\right)$.

(b) The characteristic $\mathfrak{p}_{0}^{\prime}$ of any $\varphi^{\prime}$ as in (a) is a prime ideal of $A^{\prime}$ above the characteristic $\mathfrak{p}_{0}$ of $\varphi$. 
(c) There exist a finite extension $K^{\prime} \subset K^{\mathrm{sep}}$ of $K$, a Drinfeld $A^{\prime}$-module

$$
\varphi^{\prime}: A^{\prime} \rightarrow K^{\prime}[\tau]
$$

an isogeny $f: \varphi \rightarrow \varphi^{\prime} \mid A$ over $K^{\prime}$, and an integrally closed infinite subring $B \subset A^{\prime}$ such that $A^{\prime}$ is the center of $\operatorname{End}_{K^{\operatorname{sep}}}\left(\varphi^{\prime}\right)$ and $\left(B, K^{\prime}, \varphi^{\prime} \mid B\right)$ is primitive.

(d) The subring B in (c) is unique unless $\varphi$ is isotrivial of special characteristic, in which case it is never unique.

(e) For any data as in (c) the characteristic $\mathfrak{p}_{0}^{\prime}$ of $\varphi^{\prime}$ is the unique prime ideal of $A^{\prime}$ above the characteristic $\mathfrak{q}_{0}$ of $\varphi^{\prime} \mid B$.

Proof. Applying [Devic and Pink 2012, Proposition 4.3] to $\varphi$ and the center of $\operatorname{End}_{K^{\text {sep }}}(\varphi)$ yields a Drinfeld $A$-module $\tilde{\varphi}: A \rightarrow K^{\text {sep }}[\tau]$ and an isogeny $f: \varphi \rightarrow \tilde{\varphi}$ over $K^{\text {sep }}$ such that $A^{\prime}$ is mapped into $\operatorname{End}_{K^{\text {sep }}}(\tilde{\varphi})$ under the isomorphism

$$
\operatorname{End}_{K^{\operatorname{sep}}}(\varphi) \otimes_{A} F \cong \operatorname{End}_{K^{\text {sep }}}(\tilde{\varphi}) \otimes_{A} F
$$

induced by $f$. Then $A^{\prime} \otimes_{A} F$ is the center of $\operatorname{End}_{K^{\operatorname{sep}}}(\tilde{\varphi}) \otimes_{A} F$, and since $A^{\prime}$ is integrally closed, it follows that $A^{\prime}$ is the center of $\operatorname{End}_{K^{\text {sep }}}(\tilde{\varphi})$. The tautological homomorphism $A^{\prime} \hookrightarrow \operatorname{End}_{K^{\operatorname{sep}}}(\tilde{\varphi}) \hookrightarrow K^{\operatorname{sep}}[\tau]$ thus constitutes a Drinfeld $A^{\prime}$-module $\varphi^{\prime}$ with $\varphi^{\prime} \mid A \cong \tilde{\varphi}$ and $\operatorname{End}_{K^{\operatorname{sep}}}\left(\varphi^{\prime}\right)=\operatorname{End}_{K^{\operatorname{sep}}}(\tilde{\varphi})$. In particular the center of $\operatorname{End}_{K^{\operatorname{sep}}}\left(\varphi^{\prime}\right)$ is equal to $A^{\prime}$, proving (a).

For (b) recall that the characteristic of $\varphi^{\prime}$ is the kernel of the derivative map $a^{\prime} \mapsto d \varphi_{a^{\prime}}^{\prime}$. Calling it $\mathfrak{p}_{0}^{\prime}$, the characteristic of $\varphi^{\prime} \mid A$ is then $\mathfrak{p}_{0}^{\prime} \cap A$. As the characteristic of a Drinfeld module is invariant under isogenies, it follows that $\mathfrak{p}_{0}^{\prime}$ lies above $\mathfrak{p}_{0}$, proving (b).

For the remainder of the proof we take any pair $\varphi^{\prime}$ and $f$ as in (a). We also choose a finite extension $K^{\prime} \subset K^{\text {sep }}$ of $K$ such that $\varphi^{\prime}$ and $f$ are defined over $K^{\prime}$ and that $\operatorname{End}_{K^{\prime}}\left(\varphi^{\prime}\right)=\operatorname{End}_{K^{\operatorname{sep}}}\left(\varphi^{\prime}\right)$.

Suppose first that $\varphi^{\prime}$ has generic characteristic. Then $\operatorname{End}_{K^{\prime}}\left(\varphi^{\prime}\right)$ is commutative (see [Drinfeld 1974, Corollary to Proposition 2.4]) and hence equal to $A^{\prime}$, and so the triple $\left(A^{\prime}, K^{\prime}, \varphi^{\prime}\right)$ is already primitive. This proves (c) with $B=A^{\prime}$. Also, for any integrally closed infinite subring $B \subset A^{\prime}$ the ring $\operatorname{End}_{K^{\operatorname{sep}}}\left(\varphi^{\prime} \mid B\right)$ is commutative and hence again equal to $\operatorname{End}_{K^{\operatorname{sep}}}\left(\varphi^{\prime}\right)=A^{\prime}$. Thus $\left(B, K^{\prime}, \varphi^{\prime} \mid B\right)$ being primitive requires that $B=A^{\prime}$, proving (d). Since $B=A^{\prime}$, the assertion of (e) is then trivially true.

Suppose next that $\varphi^{\prime}$ is nonisotrivial of special characteristic. Then by [Pink 2006, Theorem 6.2] there exists a unique integrally closed infinite subring $B \subset A^{\prime}$ such that $B$ is the center of $\operatorname{End}_{K^{\text {sep }}}\left(\varphi^{\prime} \mid B\right)$ and that $\operatorname{End}_{K^{\text {sep }}}\left(\varphi^{\prime} \mid B^{\prime}\right) \subset \operatorname{End}_{K^{\text {sep }}}\left(\varphi^{\prime} \mid B\right)$ for every integrally closed infinite subring $B^{\prime} \subset A^{\prime}$. For use below we note that both properties are invariant under isogenies of $\varphi^{\prime}$, because isogenies induce isomorphisms on the 
rings $\operatorname{End}_{K^{\operatorname{sep}}}\left(\varphi^{\prime} \mid B^{\prime}\right) \otimes_{B^{\prime}}$ Quot $\left(B^{\prime}\right)$. By uniqueness it follows that $B$, too, is invariant under isogenies of $\varphi^{\prime}$.

After replacing $K^{\prime}$ by a finite extension we may assume that $\operatorname{End}_{K^{\prime}}\left(\varphi^{\prime} \mid B\right)=$ $\operatorname{End}_{K^{\text {sep }}}\left(\varphi^{\prime} \mid B\right)$. Then the triple $\left(B, K^{\prime}, \varphi^{\prime} \mid B\right)$ satisfies the conditions in Definition 4.2 except that $\operatorname{End}_{K^{\prime}}\left(\varphi^{\prime} \mid B\right)$ may not be a maximal order in $\operatorname{End}_{K^{\prime}}\left(\psi^{\prime}\right) \otimes_{B} \operatorname{Quot}(B)$. But applying [Devic and Pink 2012, Proposition 4.3] to $\varphi^{\prime} \mid B$ and $\operatorname{End}_{K^{\prime}}\left(\varphi^{\prime} \mid B\right)$ yields a Drinfeld $B$-module $\psi^{\prime}: B \rightarrow K^{\prime}[\tau]$ and an isogeny $g: \varphi^{\prime} \mid B \rightarrow \psi^{\prime}$ over $K^{\prime}$ such that $\operatorname{End}_{K^{\prime}}\left(\psi^{\prime}\right)$ is a maximal order in $\operatorname{End}_{K^{\prime}}\left(\psi^{\prime}\right) \otimes_{B} \operatorname{Quot}(B)$ which contains $\operatorname{End}_{K^{\prime}}\left(\varphi^{\prime} \mid B\right)$. By the preceding remarks we now find that $\left(B, K^{\prime}, \psi^{\prime}\right)$ is primitive. Moreover, the composite homomorphism

$$
A^{\prime} \hookrightarrow \operatorname{End}_{K^{\prime}}\left(\varphi^{\prime}\right) \hookrightarrow \operatorname{End}_{K^{\prime}}\left(\varphi^{\prime} \mid B\right) \hookrightarrow \operatorname{End}_{K^{\prime}}\left(\psi^{\prime}\right) \hookrightarrow K^{\prime}[\tau]
$$

constitutes a Drinfeld $A^{\prime}$-module $\varphi^{\prime \prime}$ with $\varphi^{\prime \prime} \mid B \cong \psi^{\prime}$. After replacing $\left(\varphi^{\prime}, f\right)$ by $\left(\varphi^{\prime \prime}, g \circ f\right)$ the data then satisfies all the requirements of (c). Assertion (d) follows from the above stated uniqueness of $B$, and (e) follows from [Pink 2006, Proposition 3.5].

It remains to consider the case where $\varphi^{\prime}$ is isotrivial of special characteristic. In this case we may assume that $\varphi^{\prime}$ is defined over the constant field $k^{\prime}$ of $K^{\prime}$. Any endomorphism of $\varphi^{\prime}$ over $K^{\text {sep }}$ is then defined over a finite extension of $k^{\prime}$, but by assumption also over $K^{\prime}$; hence it is defined over $k^{\prime}$. In other words we have $\operatorname{End}_{K^{\text {sep }}}\left(\varphi^{\prime}\right) \subset k^{\prime}[\tau]$. Since $\tau^{\left[k^{\prime} / \mathbb{F}_{p}\right]}$ lies in the center of the $k^{\prime}[\tau]$, it thus corresponds to an element of the center of $\operatorname{End}_{K^{\operatorname{sep}}}\left(\varphi^{\prime}\right)$. As this center is equal to $A^{\prime}$ by assumption, there is therefore an element $a_{0} \in A^{\prime}$ with $\varphi_{a_{0}}^{\prime}=\tau^{\left[k^{\prime} / \mathbb{F}_{p}\right]}$. Set $B:=\mathbb{F}_{p}\left[a_{0}\right] \subset A^{\prime}$ which, being isomorphic to a polynomial ring, is an integrally closed infinite subring of $A^{\prime}$. Then $\operatorname{End}_{K^{\operatorname{sep}}}\left(\varphi^{\prime} \mid B\right)$ is the commutant of $\tau^{\left[k^{\prime} / \mathbb{F}_{p}\right]}$ in $K^{\text {sep }}[\tau]$ and hence just $k^{\prime}[\tau]$. By a standard construction this is a maximal $B$-order in a (cyclic) central division algebra over Quot $(B)$; hence $\left(B, K^{\prime}, \varphi^{\prime} \mid B\right)$ is primitive, proving (c). For (d) observe that replacing $K^{\prime}$ and $k^{\prime}$ by finite extensions amounts to replacing $a_{0}$ by an arbitrary positive power $a_{0}^{i}$. Thus the $\operatorname{ring} B$ is really not unique in this case, proving (d). Finally, assertion (e) follows from [Devic and Pink 2012, Proposition 6.4(a)]. This finishes the proof of Proposition 4.3.

Assume now that $(A, K, \varphi)$ is primitive and that $\varphi$ has rank $r$. Then $R \otimes_{A} F$ is a central division algebra of dimension $m^{2}$ over $F$ for some factorization $r=m n$. Thus for all primes $\mathfrak{p} \neq \mathfrak{p}_{0}$ of $A$, the ring $R_{\mathfrak{p}}:=R \otimes_{A} A_{\mathfrak{p}}$ is an $A_{\mathfrak{p}}$-order in the central simple algebra $R \otimes_{A} F_{\mathfrak{p}}$ of dimension $m^{2}$ over $F_{\mathfrak{p}}$ and is isomorphic to the matrix ring $\operatorname{Mat}_{m \times m}\left(A_{\mathfrak{p}}\right)$ for almost all $\mathfrak{p}$. Let $D_{\mathfrak{p}}$ denote the commutant of $R_{\mathfrak{p}}$ in $\operatorname{End}_{A_{\mathfrak{p}}}\left(T_{\mathfrak{p}}(\varphi)\right)$. This is an $A_{\mathfrak{p}}$-order in a central simple algebra of dimension $n^{2}$ over $F_{\mathfrak{p}}$ and is isomorphic to the matrix ring $\operatorname{Mat}_{n \times n}\left(A_{\mathfrak{p}}\right)$ for almost all $\mathfrak{p}$. Let $D_{\mathfrak{p}}^{1}$ 
denote the multiplicative group of elements of $D_{\mathfrak{p}}$ of reduced norm 1. Set

$$
D_{\mathrm{ad}}:=\prod_{\mathfrak{p} \neq \mathfrak{p}_{0}} D_{\mathfrak{p}} \subset \operatorname{End}_{A_{\mathrm{ad}}}\left(T_{\mathrm{ad}}(\varphi)\right)
$$

and

$$
D_{\mathrm{ad}}^{1}:=\prod_{\mathfrak{p} \neq \mathfrak{p}_{0}} D_{\mathfrak{p}}^{1} \subset D_{\mathrm{ad}}^{\times} \subset \operatorname{Aut}_{A_{\mathrm{ad}}}\left(T_{\mathrm{ad}}(\varphi)\right) .
$$

If $\varphi$ has generic characteristic, we have $m=1$ and therefore

$$
D_{\mathfrak{p}}=\operatorname{End}_{A_{\mathfrak{p}}}\left(T_{\mathfrak{p}}(\varphi)\right) \cong \operatorname{Mat}_{r \times r}\left(A_{\mathfrak{p}}\right)
$$

for all $\mathfrak{p}$.

If $\varphi$ is nonisotrivial of special characteristic $\mathfrak{p}_{0}$, let $a_{0}$ be any element of $A$ that generates a positive power of $\mathfrak{p}_{0}$. If $\varphi$ is isotrivial, the element $a_{0}$ from Definition 4.2(d) already has the same property. In both cases we view $a_{0}$ as a scalar element of $D_{\text {ad }}^{\times}$via the diagonal embedding $A \subset A_{\text {ad }} \subset D_{\text {ad }}$, and let $\overline{\left\langle a_{0}\right\rangle}$ denote the procyclic subgroup that is topologically generated by it.

In general the group $\Gamma_{\text {ad }}$ was described up to commensurability in our earlier work. In the primitive case, Theorem 0.1 of [Pink and Rütsche 2009] and Theorem 1.1 and Proposition 6.3 of [Devic and Pink 2012] imply:

Theorem 4.4. Assume that $(A, K, \varphi)$ is primitive.

(a) If $\varphi$ has generic characteristic, then $\Gamma_{\mathrm{ad}}$ is open in $D_{\mathrm{ad}}^{\times}$.

(b) If $\varphi$ is nonisotrivial of special characteristic, then $n \geqslant 2$ and $\Gamma_{\mathrm{ad}}$ is commensurable with $\overline{\left\langle a_{0}\right\rangle} \cdot D_{\mathrm{ad}}^{1}$.

(c) If $\varphi$ is isotrivial of special characteristic, then $n=1$ and $\Gamma_{\mathrm{ad}}=\overline{\left\langle a_{0}\right\rangle}$ with $a_{0}$ from $4.2(c)$.

Corollary 4.5. Assume that $(A, K, \varphi)$ is primitive.

(a) Let $\Theta_{\mathrm{ad}}$ denote the closure of the $\mathbb{F}_{p}$-subalgebra of $D_{\mathrm{ad}}$ generated by $\Gamma_{\mathrm{ad}}$. Then there exists a nonzero ideal $\mathfrak{a}$ of $A$ with $\mathfrak{a} \not \subset \mathfrak{p}_{0}$ such that $\mathfrak{a} D_{\mathrm{ad}} \subset \Theta_{\mathrm{ad}}$.

(b) There exist a scalar element $\gamma \in \Gamma_{\mathrm{ad}}$ and a nonzero ideal $\mathfrak{b}$ of $A$ with $\mathfrak{b} \not \subset \mathfrak{p}_{0}$ such that $\gamma \equiv 1$ modulo $\mathfrak{b} A_{\text {ad }}$ but not modulo $\mathfrak{p b} A_{\text {ad }}$ for any prime $\mathfrak{p} \neq \mathfrak{p}_{0}$ of $A$.

Proof. Any open subgroup of $D_{\text {ad }}^{\times}$, and for $n \geqslant 2$ any open subgroup of $D_{\text {ad }}^{1}$, generates an open subring of $D_{\text {ad }}$. Thus the assertion (a) follows from Theorem 4.4 unless $\varphi$ is isotrivial of special characteristic. But in that case we have $\Theta_{\mathrm{ad}}=\overline{\mathbb{F}_{p}\left[a_{0}\right]}=A_{\mathrm{ad}}=D_{\mathrm{ad}}$ and (a) follows as well.

In generic characteristic the assertion (b) follows directly from the openness of $\Gamma_{\mathrm{ad}}$. In special characteristic some positive power $a_{0}^{i}$ lies in $\Gamma_{\mathrm{ad}}$, and so (b) holds with $\gamma=a_{0}^{i}$ and the ideal $\mathfrak{b}=\left(a_{0}^{i}-1\right)$. 


\section{The primitive case}

Now we prove the following result, of which Theorem 1.6(c) is a special case:

Theorem 5.1. Assume that $(A, K, \varphi)$ is primitive. Set $R:=\operatorname{End}_{K}(\varphi)$ and let $M$ be a finitely generated torsion free $R$-submodule of $K$. Then the inclusions $\Delta_{\mathrm{ad}, M} \subset \operatorname{Hom}_{R}\left(M, T_{\mathrm{ad}}(\varphi)\right)$ and $\Gamma_{\mathrm{ad}, M} \subset \Gamma_{\mathrm{ad}} \ltimes \operatorname{Hom}_{R}\left(M, T_{\mathrm{ad}}(\varphi)\right)$ are both open.

So assume that $(A, K, \varphi)$ is primitive. Let the subring $\Theta_{\mathrm{ad}} \subset D_{\mathrm{ad}}$, the element $\gamma \in \Gamma_{\mathrm{ad}}$, and the ideals $\mathfrak{a}, \mathfrak{b} \subset A$ be as in Corollary 4.5. Since $M \subset \operatorname{Div}_{K}^{\left(\mathfrak{p}_{0}\right)}(M)$ has finite index by Theorem 2.7, we can also choose a nonzero ideal $\mathfrak{c}$ of $A$ with $\mathfrak{c} \not \subset \mathfrak{p}_{0}$ such that $\mathfrak{c} \cdot \operatorname{Div}_{K}^{\left(\mathfrak{p}_{0}\right)}(M) \subset M$. With this data we prove the following more precise version of Theorem 5.1:

Theorem 5.2. In the above situation we have $\mathfrak{a b c} \cdot \operatorname{Hom}_{R}\left(M, T_{\mathrm{ad}}(\varphi)\right) \subset \Delta_{\mathrm{ad}, M}$.

In the rest of this section we abbreviate $T_{\mathrm{ad}}:=T_{\mathrm{ad}}(\varphi)$ and $M_{\mathrm{ad}}^{*}:=\operatorname{Hom}_{R}\left(M, T_{\mathrm{ad}}\right)$. Recall that the embedding $\Delta_{\mathrm{ad}, M} \subset M_{\mathrm{ad}}^{*}$ is adjoint to the pairing $\langle$,$\rangle from (2.15).$ The arithmetic part of the proof is a calculation in $\operatorname{Div}_{K}^{\left(\mathfrak{p}_{0}\right)}(M)$ with the following result:

Lemma 5.3. For any prime $\mathfrak{p} \neq \mathfrak{p}_{0}$ of $A$ and any element $m \in M$ satisfying $\left\langle\Delta_{\mathrm{ad}, M}, m\right\rangle \subset \mathfrak{p b c} T_{\text {ad }}$ we have $m \in \mathfrak{p} M$.

Proof. The assumption on $\gamma$, viewed as an element of $A_{\text {ad }}$, means that

$$
\gamma-1 \in \mathfrak{b} A_{\text {ad }} \backslash \mathfrak{p b} A_{\text {ad }} .
$$

By the Chinese remainder theorem we can find an element $b \in A \backslash \mathfrak{p}_{0}$ satisfying $b \equiv \gamma-1$ modulo $\mathfrak{p b} \mathfrak{c} A_{\text {ad }}$. Then by construction we have $b \in \mathfrak{b} \backslash \mathfrak{p b}$, and $\gamma$ acts on all $\mathfrak{p b c}$-torsion points of $\varphi$ through the action of $1+b \in A$. By the Chinese remainder theorem we can also find elements $a, c \in A \backslash \mathfrak{p}_{0}$ with $a \in \mathfrak{p} \backslash \mathfrak{p}^{2}$ and $c \in \mathfrak{c} \backslash \mathfrak{p c}$. Then the product $a b c$ lies in $\mathfrak{p b c} \backslash\left(\mathfrak{p}^{2} \mathfrak{b} \mathfrak{c} \cup \mathfrak{p}_{0}\right)$. In particular the order of $a b c$ at $\mathfrak{p}$ is equal to that of $\mathfrak{p b c}$, and so we can also choose an element $d \in A \backslash \mathfrak{p}$ such that $d \mathfrak{p b c} \subset(a b c)$.

For better readability we abbreviate the action of any element $e \in A$ on an element $x \in K^{\text {sep }}$ by $e x:=\varphi_{e}(x)$. Since $a b c \in A \backslash \mathfrak{p}_{0}$, we can select an element $\widetilde{\tilde{m}} \in \operatorname{Div}_{K^{\text {sep }}}^{\left(\mathfrak{p}_{0}\right)}(M)$ with $a b c \widetilde{\widetilde{m}}=m$. Then $\tilde{m}:=d \widetilde{\widetilde{m}}$ is an element of $\operatorname{Div}_{K^{\text {sep }}}^{\left(\mathfrak{p}_{0}\right)}(M)$ which satisfies $a b c \tilde{m}=d m$. By construction $\tilde{m}$ lies in $K^{\text {sep }}$, but we shall see that it actually lies in a specific subfield.

Choosing a compatible system of division points of $\tilde{m}$ we can find an $A$-linear $\operatorname{map} \tilde{t}: A_{\left(\mathfrak{p}_{0}\right)} \rightarrow \operatorname{Div}_{K^{\text {sep }}}^{\left(\mathfrak{p}_{0}\right)}(M)$ satisfying $\tilde{t}\left(\frac{1}{a b c}\right)=\tilde{m}$. Then $\tilde{t}(1)=a b c \tilde{m}=d m$ lies in $M$; hence $\tilde{t}$ induces an $A$-linear map $t: A_{\left(\mathfrak{p}_{0}\right)} / A \rightarrow \operatorname{Div}_{K^{\text {sep }}}^{\left(\mathfrak{p}_{0}\right)}(M) / M$. By the construction (2.11) this map is an element of $T_{\mathrm{ad}}(\varphi, M)$ whose image in $M \otimes_{A} A_{\mathrm{ad}}$ 
is $d m \otimes 1$. For any $\delta \in \Delta_{\mathrm{ad}, M}$ the definition (2.15) of the pairing now says that $\langle\delta, d m\rangle=\delta(t)-t$. But the assumption $\left\langle\Delta_{\mathrm{ad}, M}, m\right\rangle \subset \mathfrak{p b c} T_{\mathrm{ad}}$ implies that

$$
\left\langle\Delta_{\mathrm{ad}, M}, d m\right\rangle \subset d \mathfrak{p b c} T_{\mathrm{ad}} \subset a b c T_{\mathrm{ad}}
$$

and therefore $\delta(t)-t \in a b c T_{\mathrm{ad}}$. Thus $\delta(t)-t$ is the multiple by $a b c$ of an $A$ linear map $A_{\left(\mathfrak{p}_{0}\right)} / A \rightarrow \operatorname{Div}_{K^{\text {sep }}}^{\left(\mathfrak{p}_{0}\right)}(\{0\})$; hence it is zero on the residue class of $\frac{1}{a b c}$. By the construction of $t$ this means that $\delta(\tilde{m})-\tilde{m}=0$. Varying $\delta$ we conclude that $\tilde{m}$ is fixed by $\Delta_{\mathrm{ad}, M}$; in other words, it lies in the subfield $K_{\mathrm{ad}} \subset K^{\text {sep }}$ with $\operatorname{Gal}\left(K_{\text {ad }} / K\right)=\Gamma_{\text {ad }}$.

Now consider any element $\sigma \in \operatorname{Gal}\left(K^{\mathrm{sep}} / K\right)$. The fact that $m$ lies in $K$ implies that

$$
a b c(\sigma-1)(\widetilde{\widetilde{m}})=(\sigma-1)(a b c \widetilde{\widetilde{m}})=(\sigma-1)(m)=0 .
$$

Thus $(\sigma-1)(\widetilde{\widetilde{m}})$ is annihilated by $a b c$ and hence by the ideal $d \mathfrak{p b c} \subset(a b c)$. The element $(\sigma-1)(\tilde{m})=d(\sigma-1)(\widetilde{\widetilde{m}})$ is therefore annihilated by the ideal $\mathfrak{p b c}$. Since $\gamma$ acts on all $\mathfrak{p b c}$-torsion points through the action of $1+b \in A$, it follows that

$$
(1+b-\gamma)((\sigma-1)(\tilde{m}))=0 .
$$

On the other hand we have $\tilde{m} \in K_{\text {ad }}$, and since $\gamma$ lies in the center of $\Gamma_{\text {ad }}$, its action on $K_{\text {ad }}$ commutes with the action of $\sigma$ on $K_{\text {ad }}$. Thus the last equation is equivalent to

$$
(\sigma-1)((1+b-\gamma)(\tilde{m}))=0 .
$$

As $\sigma \in \operatorname{Gal}\left(K^{\mathrm{sep}} / K\right)$ was arbitrary, it follows that $(1+b-\gamma)(\tilde{m}) \in K$.

Since $\tilde{m}$ lies in $\operatorname{Div}_{K^{\text {sep }}}^{\left(\mathfrak{p}_{0}\right)}(M)$, we can now deduce that $(1+b-\gamma)(\tilde{m}) \in \operatorname{Div}_{K}^{\left(\mathfrak{p}_{0}\right)}(M)$. By the choice of $\mathfrak{c}$ and $c$ it follows that $c(1+b-\gamma)(\tilde{m}) \in M$. The fact that $d m=a b c \tilde{m}$ thus implies that

$$
(1+b-\gamma)(d m)=(1+b-\gamma)(a b c \tilde{m})=a b c(1+b-\gamma)(\tilde{m}) \in a b M .
$$

But $d m$ is an element of $K$ and therefore satisfies $(1-\gamma)(d m)=0$. Thus the last relation shows that actually $b d m \in a b M$ and so $d m \in a M$. Since $a \in \mathfrak{p}$ and $d \notin \mathfrak{p}$, this implies that $m \in \mathfrak{p} M$, as desired.

The rest of the proof of Theorem 5.2 is a technical argument involving rings and modules. It is easier to understand if $R=A$, in which case $D_{\mathfrak{p}} \cong \operatorname{Mat}_{r \times r}\left(A_{\mathfrak{p}}\right)$ for all $\mathfrak{p}$, so the readers may want to restrict themselves to that case on first reading. For general facts on maximal orders in semisimple algebras, see [Reiner 2003].

Using Corollary 11.6 of that reference, the assumptions in Definition 4.2 imply that for any prime $\mathfrak{p} \neq \mathfrak{p}_{0}$ of $A$ the ring $R_{\mathfrak{p}}:=R \otimes_{A} A_{\mathfrak{p}}$ is a maximal order in a finite dimensional central simple algebra over $F_{\mathfrak{p}}$. By [Reiner 2003, Theorem 17.3] we can therefore identify it with the matrix ring $\operatorname{Mat}_{n_{\mathfrak{p}} \times n_{\mathfrak{p}}}\left(S_{\mathfrak{p}}\right)$, where $S_{\mathfrak{p}}$ is the maximal 
order in a finite dimensional central division algebra over $F_{\mathfrak{p}}$. Here $n_{\mathfrak{p}} \geqslant 1$ may vary with $\mathfrak{p}$. Let $L_{\mathfrak{p}}:=S_{\mathfrak{p}}^{\oplus n_{\mathfrak{p}}}$ denote the tautological left $R_{\mathfrak{p}}$-module. Since $T_{\mathfrak{p}}:=T_{\mathfrak{p}}(\varphi)$ is a nontrivial finitely generated torsion free left $R_{\mathfrak{p}}$-module, it is isomorphic to $L_{\mathfrak{p}}^{\oplus m_{\mathfrak{p}}}$ for some $m_{\mathfrak{p}} \geqslant 1$ by [ibid., Theorem 18.10]. Thus

$$
D_{\mathfrak{p}}:=\operatorname{End}_{R_{\mathfrak{p}}}\left(T_{\mathfrak{p}}\right)
$$

is isomorphic to the matrix ring $\operatorname{Mat}_{m_{\mathfrak{p}} \times m_{\mathfrak{p}}}\left(S_{\mathfrak{p}}^{\text {opp }}\right)$ over the opposite algebra $S_{\mathfrak{p}}^{\text {opp }}$. Let $N_{\mathfrak{p}}:=\left(S_{\mathfrak{p}}^{\text {opp }}\right)^{\oplus m_{\mathfrak{p}}}$ denote the tautological left $D_{\mathfrak{p}}$-module; then as a $D_{\mathfrak{p}}$-module $T_{\mathfrak{p}}$ is isomorphic to $N_{\mathfrak{p}}^{\oplus n_{\mathfrak{p}}}$. Moreover, by biduality, using Morita equivalence [ibid., Theorem 16.14] or direct computation, we have

$$
R_{\mathfrak{p}} \cong \operatorname{End}_{D_{\mathfrak{p}}}\left(T_{\mathfrak{p}}\right) .
$$

Next, since $R$ is a maximal order in a division algebra over the Dedekind ring $A$, and $M$ is a finitely generated torsion free $R$-module, $M$ is a projective $R$-module by [ibid., Corollary 21.5], say of rank $\ell \geqslant 0$. For each $\mathfrak{p} \neq \mathfrak{p}_{0}$ we therefore have $M_{\mathfrak{p}}:=M \otimes_{A} A_{\mathfrak{p}} \cong R_{\mathfrak{p}}^{\oplus \ell}$ as an $R_{\mathfrak{p}}$-module. Consequently $M_{\mathfrak{p}}^{*}:=\operatorname{Hom}_{R}\left(M, T_{\mathfrak{p}}\right) \cong T_{\mathfrak{p}}^{\oplus \ell}$ as a $D_{\mathfrak{p}}$-module via the action of $D_{\mathfrak{p}}$ on $T_{\mathfrak{p}}$. Using the biduality (5.4) we obtain a natural isomorphism

$$
M_{\mathfrak{p}} \cong \operatorname{Hom}_{D_{\mathfrak{p}}}\left(M_{\mathfrak{p}}^{*}, T_{\mathfrak{p}}\right) .
$$

Taking the product over all $\mathfrak{p} \neq \mathfrak{p}_{0}$ yields adelic versions of all this with $T_{\text {ad }}=\prod T_{\mathfrak{p}}$ and $R_{\text {ad }}=\prod R_{\mathfrak{p}}$ and $D_{\text {ad }}=\prod D_{\mathfrak{p}}$ and $M_{\text {ad }}^{*}=\prod M_{\mathfrak{p}}^{*}$.

Recall that $\Delta_{\mathrm{ad}, M}$ is a closed additive subgroup of $M_{\mathrm{ad}}^{*}=\prod M_{\mathfrak{p}}^{*}$. Let $\Delta_{\mathfrak{p}}$ denote its image under the projection to $M_{\mathfrak{p}}^{*}$.

Lemma 5.6. For any $\mathfrak{p} \neq \mathfrak{p}_{0}$ and any $D_{\mathfrak{p}}$-linear map $f: M_{\mathfrak{p}}^{*} \rightarrow N_{\mathfrak{p}}$ satisfying $f\left(\Delta_{\mathfrak{p}}\right) \subset \mathfrak{p b c} N_{\mathfrak{p}}$, we have $f\left(M_{\mathfrak{p}}^{*}\right) \subset \mathfrak{p} N_{\mathfrak{p}}$.

Proof. Since $N_{\mathfrak{p}}$ is a $D_{\mathfrak{p}}$-module isomorphic to a direct summand of $T_{\mathfrak{p}}$, it is equivalent to show that for every $D_{\mathfrak{p}}$-linear map $g: M_{\mathfrak{p}}^{*} \rightarrow T_{\mathfrak{p}}$ with $g\left(\Delta_{\mathfrak{p}}\right) \subset \mathfrak{p b c} T_{\mathfrak{p}}$ we have $g\left(M_{\mathfrak{p}}^{*}\right) \subset \mathfrak{p} T_{\mathfrak{p}}$. Let $\langle\rangle:, M_{\mathfrak{p}}^{*} \times M_{\mathfrak{p}} \rightarrow T_{\mathfrak{p}}$ denote the natural $A_{\mathfrak{p}}$-bilinear map. Then the biduality (5.5) says that $g=\left\langle_{-}, m_{\mathfrak{p}}\right\rangle$ for an element $m_{\mathfrak{p}} \in M_{\mathfrak{p}}$. Write $\mathfrak{p b} \mathfrak{c}=\mathfrak{p}^{i} \mathfrak{d}$ for an integer $i \geqslant 1$ and an ideal $\mathfrak{d}$ of $A$ that is prime to $\mathfrak{p}$. Choose any element $m \in M$ which is congruent to $m_{\mathfrak{p}}$ modulo $\mathfrak{p}^{i} M_{\mathfrak{p}}$ and congruent to 0 modulo $\mathfrak{d} M$. Then the assumption $\left\langle\Delta_{\mathfrak{p}}, m_{\mathfrak{p}}\right\rangle=g\left(\Delta_{\mathfrak{p}}\right) \subset \mathfrak{p b c} T_{\mathfrak{p}}$ implies that $\left\langle\Delta_{\mathrm{ad}, M}, m\right\rangle \subset \mathfrak{p b c} T_{\mathrm{ad}}$. By Lemma 5.3 it follows that $m \in \mathfrak{p} M$. Consequently $m_{\mathfrak{p}} \in \mathfrak{p} M_{\mathfrak{p}}$ and therefore $g\left(M_{\mathfrak{p}}^{*}\right)=\left\langle\Delta_{\mathfrak{p}}, m_{\mathfrak{p}}\right\rangle \subset \mathfrak{p} T_{\mathfrak{p}}$, as desired.

Now observe that $\Delta_{\mathrm{ad}, M} \subset M_{\mathrm{ad}}^{*}$ is a closed additive subgroup that is invariant under the action of $\Gamma_{\mathrm{ad}}$. It is therefore a submodule with respect to the subring $\Theta_{\mathrm{ad}}:=\overline{\mathbb{F}_{p}\left[\Gamma_{\mathrm{ad}}\right]}$ of $D_{\mathrm{ad}}$ from Corollary 4.5(a). By Corollary 4.5(a) we therefore 
have

$$
\Delta_{\mathrm{ad}}^{\prime}:=\mathfrak{a} D_{\mathrm{ad}} \Delta_{\mathrm{ad}, M} \subset \Delta_{\mathrm{ad}, M} .
$$

By construction $\Delta_{\text {ad }}^{\prime}$ is a submodule over $D_{\text {ad }}=\prod D_{\mathfrak{p}}$ and therefore itself a product $\Delta_{\text {ad }}^{\prime}=\prod \Delta_{\mathfrak{p}}^{\prime}$ for $D_{\mathfrak{p}}$-submodules $\Delta_{\mathfrak{p}}^{\prime} \subset M_{\mathfrak{p}}^{*}$.

Lemma 5.8. For any $\mathfrak{p} \neq \mathfrak{p}_{0}$ and any $D_{\mathfrak{p}}$-linear map $f: M_{\mathfrak{p}}^{*} \rightarrow N_{\mathfrak{p}}$ satisfying $f\left(\Delta_{\mathfrak{p}}^{\prime}\right) \subset \mathfrak{p a b c} N_{\mathfrak{p}}$, we have $f\left(M_{\mathfrak{p}}^{*}\right) \subset \mathfrak{p} N_{\mathfrak{p}}$.

Proof. The definition of $\Delta_{\mathfrak{p}}^{\prime}$ implies that $\mathfrak{a} D_{\mathfrak{p}} \Delta_{\mathfrak{p}} \subset \Delta_{\mathfrak{p}}^{\prime}$. Thus by assumption we have $\mathfrak{a} f\left(\Delta_{\mathfrak{p}}\right) \subset f\left(\mathfrak{a} D_{\mathfrak{p}} \Delta_{\mathfrak{p}}\right) \subset f\left(\Delta_{\mathfrak{p}}^{\prime}\right) \subset \mathfrak{p a b} \mathfrak{c} N_{\mathfrak{p}}$ and therefore $f\left(\Delta_{\mathfrak{p}}\right) \subset \mathfrak{p b} \mathfrak{c} N_{\mathfrak{p}}$. By Lemma 5.6 this implies that $f\left(M_{\mathfrak{p}}^{*}\right) \subset \mathfrak{p} N_{\mathfrak{p}}$.

Lemma 5.9. For any $\mathfrak{p} \neq \mathfrak{p}_{0}$ we have $\mathfrak{a b c} M_{\mathfrak{p}}^{*} \subset \Delta_{\mathfrak{p}}^{\prime}$.

Proof. Let $\mathfrak{m}_{\mathfrak{p}}$ denote the maximal ideal of $S_{\mathfrak{p}}^{\text {opp }}$. Then by [Reiner 2003, Theorem 13.2] we have $\mathfrak{p} S_{\mathfrak{p}}^{\text {opp }}=\mathfrak{m}_{\mathfrak{p}}^{e}$ for some integer $e \geqslant 1$. The general theory says the following about the structure of the module $M_{\mathfrak{p}}^{*} / \Delta_{\mathfrak{p}}^{\prime}$ over the maximal order $D_{\mathfrak{p}}$. On the one hand, by [Knebusch 1967, Satz 7] the torsion submodule of $M_{\mathfrak{p}}^{*} / \Delta_{\mathfrak{p}}^{\prime}$ is a finite direct sum of indecomposable modules isomorphic to $N_{\mathfrak{p}} / \mathfrak{m}_{\mathfrak{p}}^{j_{v}} N_{\mathfrak{p}}$ for certain integers $j_{v} \geqslant 1$. On the other hand, the factor module of $M_{\mathfrak{p}}^{*} / \Delta_{\mathfrak{p}}^{\prime}$ by its torsion submodule is projective by [Reiner 2003, Corollary 21.5] and hence isomorphic to a direct sum of copies of $N_{\mathfrak{p}}$. That the factor module is projective also implies that $M_{\mathfrak{p}}^{*} / \Delta_{\mathfrak{p}}^{\prime}$ is isomorphic to the direct sum of its torsion submodule with the factor module. Together it follows that $M_{\mathfrak{p}}^{*} / \Delta_{\mathfrak{p}}^{\prime}$ is a finite direct sum of modules isomorphic to $N_{\mathfrak{p}}$ or to $N_{\mathfrak{p}} / \mathfrak{m}_{\mathfrak{p}}^{j_{\mathfrak{v}}} N_{\mathfrak{p}}$ for certain integers $j_{v} \geqslant 1$.

To use this fact, let $\mathfrak{p}^{i}$ denote the highest power of $\mathfrak{p}$ dividing $\mathfrak{a} \mathfrak{b} \mathfrak{c}$. If no summand isomorphic to $N_{\mathfrak{p}}$ occurs in $M_{\mathfrak{p}}^{*} / \Delta_{\mathfrak{p}}^{\prime}$ and all exponents $j_{\nu}$ are $\leqslant e i$, then $M_{\mathfrak{p}}^{*} / \Delta_{\mathfrak{p}}^{\prime}$ is annihilated by $\mathfrak{p}^{i} S_{\mathfrak{p}}^{\text {opp }}=\mathfrak{m}_{\mathfrak{p}}^{e i}$. In this case it follows that $\mathfrak{a b c} M_{\mathfrak{p}}^{*}=\mathfrak{p}^{i} M_{\mathfrak{p}}^{*} \subset \Delta_{\mathfrak{p}}^{\prime}$, as desired.

Otherwise there exists a surjective $D_{\mathfrak{p}}$-linear map $M_{\mathfrak{p}}^{*} / \Delta_{\mathfrak{p}}^{\prime} \rightarrow N_{\mathfrak{p}} / \mathfrak{m}_{\mathfrak{p}}^{e i+1} N_{\mathfrak{p}}$. Composed with the isomorphism

$$
N_{\mathfrak{p}} / \mathfrak{m}_{\mathfrak{p}}^{e i+1} N_{\mathfrak{p}} \cong \mathfrak{m}_{\mathfrak{p}}^{e-1} N_{\mathfrak{p}} / \mathfrak{p}^{i+1} N_{\mathfrak{p}}=\mathfrak{m}_{\mathfrak{p}}^{e-1} N_{\mathfrak{p}} / \mathfrak{p a b c} N_{\mathfrak{p}},
$$

this yields a $D_{\mathfrak{p}}$-linear map $M_{\mathfrak{p}}^{*} / \Delta_{\mathfrak{p}}^{\prime} \rightarrow N_{\mathfrak{p}} / \mathfrak{p a b c} N_{\mathfrak{p}}$ whose image is not contained in $\mathfrak{p} N_{\mathfrak{p}} / \mathfrak{p} \mathfrak{a b} \mathfrak{c} N_{\mathfrak{p}}=\mathfrak{m}_{\mathfrak{p}}^{e} N_{\mathfrak{p}} / \mathfrak{p} \mathfrak{a b} \mathfrak{c} N_{\mathfrak{p}}$. As $M_{\mathfrak{p}}^{*}$ is a projective $D_{\mathfrak{p}}$-module, the latter map can be lifted to a $D_{\mathfrak{p}}$-linear map $f: M_{\mathfrak{p}}^{*} \rightarrow N_{\mathfrak{p}}$. By construction this map then satisfies $f\left(\Delta_{\mathfrak{p}}^{\prime}\right) \subset \mathfrak{p a b c} N_{\mathfrak{p}}$ and $f\left(M_{\mathfrak{p}}^{*}\right) \not \subset \mathfrak{p} N_{\mathfrak{p}}$. But that contradicts Lemma 5.8; hence this case is not possible and the lemma is proved.

Taking the product over all $\mathfrak{p}$, Lemma 5.9 and the inclusion (5.7) imply that $\mathfrak{a b} \mathfrak{c} M_{\text {ad }}^{*} \subset \Delta_{\text {ad }}^{\prime} \subset \Delta_{\mathrm{ad}, M}$. This finishes the proof of Theorem 5.2. In particular it proves the first assertion of Theorem 5.1, from which the second assertion directly follows. 


\section{The general case}

First we note the following general fact on homomorphisms of modules:

Proposition 6.1. Let $S$ be a unitary ring, not necessarily commutative, and let $M$ and $N$ be left $S$-modules. Let $X$ be a subset of $M$ and $S X$ the $S$-submodule generated by it. Let $\operatorname{Hom}_{(S)}(X, N)$ denote the set of maps $\ell: X \rightarrow N$ such that for any finite collection of $s_{i} \in S$ and $x_{i} \in X$ with $\sum_{i} s_{i} x_{i}=0$ in $M$ we have $\sum_{i} s_{i} \ell\left(x_{i}\right)=0$ in $N$.

(a) The restriction of maps induces a bijection $\operatorname{Hom}_{S}(S X, N) \stackrel{\sim}{\rightarrow} \operatorname{Hom}_{(S)}(X, N)$.

(b) If $R$ is a unitary subring of $S$ such that $X$ is an $R$-submodule and the natural map $S \otimes_{R} X \rightarrow M, \sum_{i} s_{i} \otimes x_{i} \mapsto \sum_{i} s_{i} x_{i}$ is injective, then $\operatorname{Hom}_{(S)}(X, N)=$ $\operatorname{Hom}_{R}(X, N)$.

(c) If $X$ is an $S$-submodule of $M$, then $\operatorname{Hom}_{(S)}(X, N)=\operatorname{Hom}_{S}(X, N)$.

Proof. Let $F:=\bigoplus_{x \in X} S \cdot[x]$ be the free left $S$-module over the set $X$ and consider the natural $S$-linear map $F \rightarrow M, \sum_{i} s_{i}\left[x_{i}\right] \mapsto \sum_{i} s_{i} x_{i}$. Since $S$ is unitary, the image of this map is $S X$. Let $T$ denote its kernel. Then giving an $S$-linear map $S X \rightarrow N$ is equivalent to giving an $S$-linear map $F \rightarrow N$ which vanishes on $T$. Using the universal property of $F$ we find that the latter is equivalent to giving an element of $\operatorname{Hom}_{(S)}(X, N)$. The total correspondence is given by restriction of maps, proving (a).

In (b) we have $S \otimes_{R} X \stackrel{\sim}{\rightarrow} S X$; hence the adjunction between tensor product and Hom yields bijections $\operatorname{Hom}_{S}(S X, N) \stackrel{\sim}{\rightarrow} \operatorname{Hom}_{S}\left(S \otimes_{R} X, N\right) \stackrel{\sim}{\rightarrow} \operatorname{Hom}_{R}(X, N)$. Their composite is again just restriction of maps; so by (a) the restriction map $\operatorname{Hom}_{(S)}(X, N) \rightarrow \operatorname{Hom}_{R}(X, N)$ is also bijective, proving (b).

Finally, (c) is a special case of (a) or (b), according to taste.

Now we return to the situation of Section 4 . We choose data $\left(A^{\prime}, K^{\prime}, \varphi^{\prime}, f, B\right)$ as in Proposition 4.3(c), that is: We let $A^{\prime}$ denote the normalization of the center of $\operatorname{End}_{K^{\operatorname{sep}}}(\varphi)$, take a finite extension $K^{\prime} \subset K^{\text {sep }}$ of $K$, a Drinfeld $A^{\prime}$-module $\varphi^{\prime}: A^{\prime} \rightarrow K^{\prime}[\tau]$, an isogeny $f: \varphi \rightarrow \varphi^{\prime} \mid A$ over $K^{\prime}$, and an integrally closed infinite subring $B \subset A^{\prime}$ such that $A^{\prime}$ is the center of $\operatorname{End}_{K^{\operatorname{sep}}}\left(\varphi^{\prime}\right)$ and $\left(B, K^{\prime}, \varphi^{\prime} \mid B\right)$ is primitive. By Proposition 4.3(e) the characteristic $\mathfrak{p}_{0}^{\prime}$ of $\varphi^{\prime}$ is then the only prime ideal of $A^{\prime}$ above the characteristic $\mathfrak{q}_{0}$ of $\varphi^{\prime} \mid B$. We will apply the reduction steps from Section 3 to the isogeny $f$ and to each of the inclusions $A \subset A^{\prime} \supset B$.

Specifically, let us set $\psi^{\prime}:=\varphi^{\prime} \mid B$ and $S^{\prime}:=\operatorname{End}_{K^{\operatorname{sep}}}\left(\psi^{\prime}\right)$. Then $M^{\prime}:=A^{\prime} f(M)$ is a finitely generated $A^{\prime}$-submodule of $K^{\prime}$ for the action of $A^{\prime}$ through $\varphi^{\prime}$, and so $N^{\prime}:=S^{\prime} M^{\prime}$ is a finitely generated $B$-submodule for the action of $B$ through $\psi^{\prime}$. The modules $M^{\prime}$ and $N^{\prime}$ may have torsion, but since they are finitely generated, their torsion is annihilated by some nonzero element $a^{\prime} \in A^{\prime}$. Replacing the isogeny 
$f$ by $\varphi_{a^{\prime}}^{\prime} \circ f$ replaces $M^{\prime}$ by $a^{\prime} M^{\prime}$ and $N^{\prime}$ by $a^{\prime} N^{\prime}$; hence we may without loss of generality assume that $M^{\prime}$ and $N^{\prime}$ are torsion free.

Let $\Delta_{\mathrm{ad}, M^{\prime}}^{\varphi^{\prime}} \subset \Gamma_{\mathrm{ad}, M^{\prime}}^{\varphi^{\prime}} \rightarrow \Gamma_{\mathrm{ad}}^{\varphi^{\prime}}$ denote the Galois groups as in (2.14) associated to $\left(K^{\prime}, \varphi^{\prime}, M^{\prime}\right)$ in place of $(K, \varphi, M)$, and similarly for $\left(K^{\prime}, \varphi^{\prime}, N^{\prime}\right)$, respectively for $\left(K^{\prime}, \psi^{\prime}, N^{\prime}\right)$, and so on. Then Proposition 3.5 for the inclusion $A^{\prime} \supset B$ yields a natural commutative diagram with exact rows

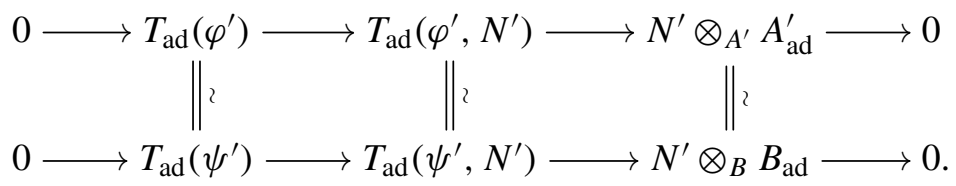

The action of $S^{\prime}$ on the lower row thus yields a natural action on the upper row. Recall from (2.19) that any $S^{\prime}$-equivariant splitting induces an embedding

$$
\Gamma_{\mathrm{ad}, N^{\prime}}^{\psi^{\prime}} \hookrightarrow \Gamma_{\mathrm{ad}}^{\psi^{\prime}} \ltimes \operatorname{Hom}_{S^{\prime}}\left(N^{\prime}, T_{\mathrm{ad}}\left(\psi^{\prime}\right)\right) .
$$

Since $\left(B, K^{\prime}, \psi^{\prime}\right)$ is primitive, this embedding is open by Theorem 5.1. The isomorphisms from (3.6) thus yield an open embedding

$$
\Gamma_{\mathrm{ad}, N^{\prime}}^{\varphi^{\prime}} \hookrightarrow \Gamma_{\mathrm{ad}}^{\varphi^{\prime}} \ltimes \operatorname{Hom}_{S^{\prime}}\left(N^{\prime}, T_{\mathrm{ad}}\left(\varphi^{\prime}\right)\right) .
$$

Since $N^{\prime}=S^{\prime} M^{\prime}$, the Galois action on $T_{\text {ad }}\left(\varphi^{\prime}, N^{\prime}\right)$ is completely determined by the action on $T_{\mathrm{ad}}\left(\varphi^{\prime}, M^{\prime}\right)$; in other words the restriction induces a natural isomorphism $\Gamma_{\mathrm{ad}, N^{\prime}}^{\varphi^{\prime}} \cong \Gamma_{\mathrm{ad}, M^{\prime}}^{\varphi^{\prime}}$. This together with Proposition 6.1(a) yields an open embedding

$$
\Gamma_{\mathrm{ad}, M^{\prime}}^{\varphi^{\prime}} \hookrightarrow \Gamma_{\mathrm{ad}}^{\varphi^{\prime}} \ltimes \operatorname{Hom}_{\left(S^{\prime}\right)}\left(M^{\prime}, T_{\mathrm{ad}}\left(\varphi^{\prime}\right)\right) .
$$

The next natural step would be the passage from $\left(K^{\prime}, \varphi^{\prime}, M^{\prime}\right)$ to $\left(K^{\prime}, \varphi^{\prime} \mid A, M^{\prime}\right)$. However, this runs into the problem that $S^{\prime}$ does not necessarily act on $T_{\text {ad }}\left(\varphi^{\prime} \mid A\right)$, because $T_{\text {ad }}\left(\varphi^{\prime} \mid A\right)$ is obtained from $T_{\text {ad }}\left(\varphi^{\prime}\right) \cong \prod_{\mathfrak{p}^{\prime} \neq \mathfrak{p}_{0}^{\prime}} T_{\mathfrak{p}^{\prime}}\left(\varphi^{\prime}\right)$ by removing all factors with $\mathfrak{p}^{\prime} \mid \mathfrak{p}_{0}^{\prime}$, which are not necessarily preserved by the noncommutative ring $S^{\prime}$. Thus if $\mathfrak{p}_{0}^{\prime}$ is not the only prime above $\mathfrak{p}_{0}$, it would be ugly to precisely describe the image of $\operatorname{Hom}_{\left(S^{\prime}\right)}\left(M^{\prime}, T_{\mathrm{ad}}\left(\varphi^{\prime}\right)\right)$ in $\operatorname{Hom}_{A}\left(M^{\prime}, T_{\mathrm{ad}}\left(\varphi^{\prime} \mid A\right)\right)$ in general, though of course it can be done. We therefore restrict ourselves to two special cases, with the following results:

Theorem 6.6. Assume that $A$ is the center of $\operatorname{End}_{K^{\operatorname{sep}}}(\varphi)$. With $A^{\prime}:=A$ let $\left(K^{\prime}, \varphi^{\prime}, f, B\right)$ be as in Proposition 4.3(c), and set $S:=\operatorname{End}_{K^{\operatorname{sep}}}(\varphi \mid B)$. Let $M$ be a finitely generated torsion free A-submodule of $K$. Then $\Delta_{\mathrm{ad}, M}$ is commensurable with the subgroup $\operatorname{Hom}_{(S)}\left(M, T_{\mathrm{ad}}(\varphi)\right)$ of $\operatorname{Hom}_{A}\left(M, T_{\mathrm{ad}}(\varphi)\right)$, and $\Gamma_{\mathrm{ad}, M}$ is commensurable with $\Gamma_{\text {ad }} \ltimes \operatorname{Hom}_{(S)}\left(M, T_{\text {ad }}(\varphi)\right)$.

Proof. With the above notation $f$ induces an isomorphism $M \stackrel{\sim}{\rightarrow} M^{\prime}$ and an embedding of finite index $T_{\mathrm{ad}}(\varphi) \hookrightarrow T_{\mathrm{ad}}\left(\varphi^{\prime}\right)$. It also induces an isomorphism 
$S \otimes_{B}$ Quot $(B) \cong S^{\prime} \otimes_{B}$ Quot $(B)$ under which the intersection of $S$ and $S^{\prime}$ has finite index in both. Since $T_{\mathrm{ad}}(\varphi)$ and $T_{\mathrm{ad}}\left(\varphi^{\prime}\right)$ are torsion free $A_{\mathrm{ad}}$-modules, this implies the equalities and an inclusion of finite index in the diagram

$$
\begin{gathered}
\operatorname{Hom}_{(S)}\left(M, T_{\mathrm{ad}}(\varphi)\right)=\operatorname{Hom}_{\left(S \cap S^{\prime}\right)}\left(M, T_{\mathrm{ad}}(\varphi)\right) \\
\downarrow_{f \circ() \circ f^{-1}} f\left(M^{\prime}, T_{\mathrm{ad}}\left(\varphi^{\prime}\right)\right) . \\
\operatorname{Hom}_{\left(S^{\prime}\right)}\left(M^{\prime}, T_{\mathrm{ad}}\left(\varphi^{\prime}\right)\right)=\operatorname{Hom}_{\left(S \cap S^{\prime}\right)}\left(M^{\prime}\right)
\end{gathered}
$$

With (3.2) and the open embedding (6.5) this shows that the image of $\mathrm{Gal}\left(K^{\mathrm{sep}} / K^{\prime}\right)$ associated to $\left(K^{\prime}, \varphi, M\right)$ is an open subgroup of $\Gamma_{\mathrm{ad}} \ltimes \operatorname{Hom}_{(S)}\left(M, T_{\mathrm{ad}}(\varphi)\right)$. This implies the assertion about $\Gamma_{\mathrm{ad}, M}$, from which the assertion about $\Delta_{\mathrm{ad}, M}$ directly follows.

In the other special case we drop all assumptions on endomorphisms, but instead assume something about $M$ :

Theorem 6.7. Let $\left(A^{\prime}, K^{\prime}, \varphi^{\prime}, f, B\right)$ be as in Proposition 4.3(c), and set

$$
S^{\prime}:=\operatorname{End}_{K^{\operatorname{sep}}}\left(\varphi^{\prime} \mid B\right) \text {. }
$$

Let $M$ be a finitely generated torsion free A-submodule of $K$ such that the natural map

$$
S^{\prime} \otimes_{A} M \rightarrow K^{\mathrm{sep}}, \quad \sum_{i} s_{i} \otimes m_{i} \mapsto \sum_{i} s_{i} f\left(m_{i}\right)
$$

is injective. Then $\Delta_{\mathrm{ad}, M}$ is an open subgroup of $\operatorname{Hom}_{A}\left(M, T_{\mathrm{ad}}(\varphi)\right)$, and $\Gamma_{\mathrm{ad}, M}$ is an open subgroup of $\Gamma_{\mathrm{ad}} \ltimes \operatorname{Hom}_{A}\left(M, T_{\mathrm{ad}}(\varphi)\right)$.

Proof. With the above notation the assumption implies that the natural map

$$
S^{\prime} \otimes_{A} f(M) \rightarrow S^{\prime} f(M)=N^{\prime}, \quad \sum_{i} s_{i}^{\prime} \otimes m_{i}^{\prime} \mapsto \sum_{i} s_{i}^{\prime} m_{i}^{\prime}
$$

is injective and therefore an isomorphism. Thus by Proposition 6.1 the restriction induces a natural isomorphism

$$
\operatorname{Hom}_{S^{\prime}}\left(N^{\prime}, T_{\mathrm{ad}}\left(\varphi^{\prime}\right)\right) \stackrel{\sim}{\rightarrow} \operatorname{Hom}_{A}\left(f(M), T_{\mathrm{ad}}\left(\varphi^{\prime}\right)\right) .
$$

The openness of the embedding (6.4), together with the surjectivity in (3.6) for the inclusion $A \subset A^{\prime}$, thus implies the openness of the embedding

$$
\Gamma_{\mathrm{ad}, f(M)}^{\varphi^{\prime} \mid A} \hookrightarrow \Gamma_{\mathrm{ad}}^{\varphi^{\prime} \mid A} \ltimes \operatorname{Hom}_{A}\left(f(M), T_{\mathrm{ad}}\left(\varphi^{\prime} \mid A\right)\right) .
$$

With (3.2) it follows that

$$
\Gamma_{\mathrm{ad}, M} \hookrightarrow \Gamma_{\mathrm{ad}} \ltimes \operatorname{Hom}_{A}\left(M, T_{\mathrm{ad}}(\varphi)\right)
$$

is an open embedding. This proves the assertion about $\Gamma_{\mathrm{ad}, M}$, from which the assertion about $\Delta_{\mathrm{ad}, M}$ directly follows. 


\section{References}

[Chi and Li 2001] W.-C. Chi and A. Li, "Kummer theory of division points over Drinfeld modules of rank one", J. Pure Appl. Algebra 156:2-3 (2001), 171-185. MR 2001k:11233 Zbl 1029.11018

[Deligne and Husemöller 1987] P. Deligne and D. Husemöller, "Survey of Drinfel'd modules", pp. 25-91 in Current trends in arithmetical algebraic geometry (Arcata, California, 1985), edited by K. A. Ribet, Contemp. Math. 67, Amer. Math. Soc., Providence, RI, 1987. MR 89f:11081

[Devic and Pink 2012] A. Devic and R. Pink, “Adelic openness for Drinfeld modules in special characteristic", J. Number Theory 132:7 (2012), 1583-1625. MR 2903172 Zbl 06033752

[Drinfeld 1974] V. G. Drinfel'd, "Elliptic modules", Mat. Sb. (N.S.) 94:136 (1974), 594-627, 656. In Russian; translated in Math. USSR Sbornik 23:4 (1974), 561-592. MR 52 \#5580

[Goss 1996] D. Goss, Basic structures of function field arithmetic, Ergebnisse der Mathematik und ihrer Grenzgebiete (3) 35, Springer, Berlin, 1996. MR 97i:11062 Zbl 0874.11004

[Häberli 2011] S. Häberli, Kummer theory of Drinfeld modules, Master's thesis, ETH Zürich, 2011, Available at http://www.math.ethz.ch/ pink/Theses/2011-Master-Simon-Haeberli.pdf.

[Hayes 1979] D. R. Hayes, "Explicit class field theory in global function fields", pp. 173-217 in Studies in algebra and number theory, edited by G.-C. Rota, Adv. in Math. Suppl. Stud. 6, Academic Press, New York, 1979. MR 81d:12011 Zbl 0476.12010

[Knebusch 1967] M. Knebusch, "Elementarteilertheorie über Maximalordnungen", J. Reine Angew. Math. 226 (1967), 175-183. MR 35 \#5433 Zbl 0217.33704

[Li 2001] A. Li, "A note on Kummer theory of division points over singular Drinfeld modules", Bull. Austral. Math. Soc. 64:1 (2001), 15-20. MR 2002f:11065 Zbl 0984.11026

[Pink 2006] R. Pink, "The Galois representations associated to a Drinfeld module in special characteristic, II: Openness", J. Number Theory 116:2 (2006), 348-372. MR 2006k:11108 Zbl 1173.11037

[Pink and Rütsche 2009] R. Pink and E. Rütsche, "Adelic openness for Drinfeld modules in generic characteristic”, J. Number Theory 129:4 (2009), 882-907. MR 2010f:11092 Zbl 1246.11122

[Poonen 1995] B. Poonen, "Local height functions and the Mordell-Weil theorem for Drinfel'd modules", Compositio Math. 97:3 (1995), 349-368. MR 96k:11075

[Reiner 2003] I. Reiner, Maximal orders, London Mathematical Society Monographs. New Series 28, The Clarendon Press, Oxford University Press, Oxford, 2003. Corrected reprint of the 1975 original. MR 2004c:16026 Zbl 1024.16008

[Ribet 1979] K. A. Ribet, "Kummer theory on extensions of abelian varieties by tori”, Duke Math. J. 46:4 (1979), 745-761. MR 81g:14019 Zbl 0428.14018

[Wang 2001] J. T.-Y. Wang, "The Mordell-Weil theorems for Drinfeld modules over finitely generated function fields”, Manuscripta Math. 106:3 (2001), 305-314. MR 2002k:11086 Zbl 0992.11039

Communicated by Bjorn Poonen

Received 2012-02-21 Revised 2012-07-11 Accepted 2012-11-05

pink@math.ethz.ch Department of Mathematics, ETH Zürich, CH-8092 Zürich, Switzerland 


\section{Algebra \& Number Theory}

msp.org/ant

\section{EDITORS}

MANAGING EDITOR

Bjorn Poonen

Massachusetts Institute of Technology

Cambridge, USA

\author{
EDITORIAL BOARD CHAIR \\ David Eisenbud \\ University of California \\ Berkeley, USA
}

BOARD OF EDITORS

Georgia Benkart

Dave Benson

Richard E. Borcherds

John H. Coates

J-L. Colliot-Thélène

Brian D. Conrad

Hélène Esnault

Hubert Flenner

Sergey Fomin

Edward Frenkel

Andrew Granville

Joseph Gubeladze

Roger Heath-Brown

Craig Huneke

Kiran S. Kedlaya

János Kollár

Yuri Manin

Philippe Michel
University of Wisconsin, Madison, USA

University of Aberdeen, Scotland

University of California, Berkeley, USA

University of Cambridge, UK

CNRS, Université Paris-Sud, France

Stanford University, USA

Freie Universität Berlin, Germany

Ruhr-Universität, Germany

University of Michigan, USA

University of California, Berkeley, USA

Université de Montréal, Canada

San Francisco State University, USA

Oxford University, UK

University of Virginia, USA

Univ. of California, San Diego, USA

Princeton University, USA

Northwestern University, USA

École Polytechnique Fédérale de Lausanne
Susan Montgomery

Shigefumi Mori

Raman Parimala

Jonathan Pila

Anand Pillay

Victor Reiner

Peter Sarnak

Joseph H. Silverman

Michael Singer

Vasudevan Srinivas

J. Toby Stafford

Ravi Vakil

Michel van den Bergh

Marie-France Vignéras

Kei-Ichi Watanabe

Efim Zelmanov

Shou-Wu Zhang
University of Southern California, USA

RIMS, Kyoto University, Japan

Emory University, USA

University of Oxford, UK

University of Notre Dame, USA

University of Minnesota, USA

Princeton University, USA

Brown University, USA

North Carolina State University, USA

Tata Inst. of Fund. Research, India

University of Michigan, USA

Stanford University, USA

Hasselt University, Belgium

Université Paris VII, France

Nihon University, Japan

University of California, San Diego, USA

Princeton University, USA

\section{PRODUCTION}

production@msp.org

Silvio Levy, Scientific Editor

See inside back cover or msp.org/ant for submission instructions.

The subscription price for 2016 is US $\$ 290 /$ year for the electronic version, and $\$ 485 /$ year ( $+\$ 55$, if shipping outside the US) for print and electronic. Subscriptions, requests for back issues and changes of subscribers address should be sent to MSP.

Algebra \& Number Theory (ISSN 1944-7833 electronic, 1937-0652 printed) at Mathematical Sciences Publishers, 798 Evans Hall \#3840, c/o University of California, Berkeley, CA 94720-3840 is published continuously online. Periodical rate postage paid at Berkeley, CA 94704, and additional mailing offices.

ANT peer review and production are managed by EditFLOW ${ }^{\circledR}$ from MSP.

\section{PUBLISHED BY}

- mathematical sciences publishers

nonprofit scientific publishing

http://msp.org/

(C) 2016 Mathematical Sciences Publishers 


\section{Algebra \& Number Theory}

Volume $10 \quad$ No. $2 \quad 2016$

Kummer theory for Drinfeld modules

RICHARD PINK

Parity and symmetry in intersection and ordinary cohomology

SHENGHAO SUN and WeIZHE ZHENG

DANIEL KRIZ

Squarefree polynomials and Möbius values in short intervals and arithmetic progressions JONATHAN P. KEATING and ZEEV RUDNICK

Equidistribution of values of linear forms on a cubic hypersurface SAM CHOW 